\title{
Magnetic oscillations in planar systems with the Dirac-like spectrum of quasiparticle excitations
}

\author{
S.G. Sharapov ${ }^{1}$ V.P. Gusynin ${ }^{2}$ 田 and H. Beck团 \\ ${ }^{1}$ Institut de Physique, Université de Neuchâtel, 2000 Neuchâtel, Switzerland \\ ${ }^{2}$ Department of Applied Mathematics, University of Western Ontario, London, Ontario N6A 5B7, Canada
}

(Dated: October 31, 2018)

\begin{abstract}
The quantum magnetic oscillations are studied for planar condensed matter systems with a linear, Dirac-like spectrum of quasiparticle excitations. We derive analytical expressions for magnetic oscillations (de Haas - van Alphen effect) in the density of states, thermodynamic potential, magnetization, and chemical potential both for zero and finite temperatures, and in the presence of scattering from impurities. We discuss also a possibility of using magnetic oscillations for detection of a gap that may open in the spectrum of quasiparticle excitations due to a nontrivial interaction between them.

PACS numbers: 71.10.-w, 71.70.Di, 71.18.+y, 11.10.Wx
\end{abstract}

\section{INTRODUCTION}

Magnetic oscillations (MO) in metals [1] proved to be a powerful tool for investigation of the shape of their Fermi surface. The oscillations of magnetization were predicted by Landau in 1930 [2]. The experimental discovery of MO came soon: Shubnikov and de Haas $(\mathrm{SdH})$ found oscillations of electrical conductivity in Bi crystals, and later de Haas and van Alphen ( $\mathrm{dHvA}$ ) discovered the oscillations of magnetization. Despite more than 70 years old history of MO they continue to attract attention of both experimentalists and theorists. The recent experimental studies are mostly focused on quasi-two-dimensional (quasi-2D) organic conductors and superconductors (for a review, see e.g. 3]). The ultimate hope is that better understanding of the $2 \mathrm{D}$ and quasi-2D organic systems can also contribute to the studies of high-temperature superconductors that have a similar layered structure. As suggested recently in Ref. 4], dHvA experiment can be used as a probe to detect band- and/or angle-dependent gap amplitudes of type-II superconductors.

It turns out that these experimental advances demand also further development of the MO theory. While MO observed in 3D metals are well described by Lifshits-Kosevich (LK) [5] (for dHvA effect) and Adams-Holstein 6] (for $\mathrm{SdH}$ effect) theories, there is no commonly accepted and used theory for $\mathrm{MO}$ in $2 \mathrm{D}$ and quasi-2D materials $[$ ]. As discussed in Ref. 7], there are two essential features of dHvA effect in 2D case that differ it from 3D case:

(1) The sharp saw-tooth like shape of the oscillations seen in the low temperature $T \ll \omega_{L}$ and high purity $\Gamma \ll \omega_{L}$ regions. Here, $\omega_{L}$ is the distance between Landau levels and $\Gamma$ is the width of Landau's levels due to impurity scattering.

(2) Due to the Landau quantization of the 2D kinetic energy in the magnetic field, both the density of electrons, $n$, and the chemical potential, $\mu$, cannot be fixed simultaneously as the magnetic field is changed. Depending on the physical situation, two extreme limits are usually considered:

(i) the limit of fixed and field independent chemical potential $\mu$, which can be represented by a grand canonical ensemble. This corresponds to the case originally studied by Lifshits and Kosevich [5] in 3D.

(ii) the limit of fixed density of carriers $n$, in which a strong dependence of the chemical potential $\mu$ on the magnetic field that has to be taken into account by considering an equation for $\mu$ that leads to the chemical potential oscillations. This equation is crucial in 2D case even for the large Fermi energy, $\epsilon_{F} \gg \omega_{L}$ since despite smallness of the chemical potential shift, $\delta \mu \sim \omega_{L}$ due to the magnetic field compared to $\epsilon_{F}$, it can change the phase of the magnetization MO (in 3D the oscillating part $\delta \mu \sim \omega_{L}\left(\omega_{L} / \epsilon_{F}\right)^{1 / 2}$, so that for $\epsilon_{F} \gg \omega_{L}$ it does not alter the MO with a high accuracy). Nevertheless, under certain conditions discussed in Refs. 7, $[8]$ the oscillations of the chemical potential are small and one may still assume that the chemical potential $\mu$ coincides with the Fermi energy $\epsilon_{F}$ as in the limit (i).

*Electronic address: sharapov@isiosf.isi.it Present address: Institute for Scientific Interchange, via Settimio Severo 65, I-10133 Torino, Italy

${ }^{\dagger}$ On leave of absence from Bogolyubov Institute for Theoretical Physics, Metrologicheskaya Str. 14-b, Kiev, 03143, Ukraine; Electronic address: vgusynin@bitp.kiev.ua

‡Electronic address: Hans.Beck@unine.ch URL: http://www.unine.ch/phys/theocond/ 
On the other hand, in condensed matter systems with a linear, "relativistic" spectrum of quasiparticle excitations, the conditions favorable for MO seem to persist to rather small magnetic fields and high temperatures [9]. This is particularly true for 2D systems with the Dirac quasiparticle spectrum.

There is a wide variety of planar condensed matter systems that in the low-energy limit have a linear dispersion law of the quasiparticle excitations. In particular, the studies of high-temperature cuprate superconductors inspired a big interest in the so-called nodal excitations that are the gapless fermion excitations associated with zeros of the gap function. Depending on the physical origin of the gap, one can consider rather different physical situations that are in general described by different theoretical models. For example, if the gap opens due to the anisotropic electron-hole pairing, which is one of the possible states of the electron system with a half-filled band and nested Fermi surface, this corresponds to a 2D orbital antiferromagnet (OAF) or staggered flux state [10, 11, 12].

There is a consensus that the superconducting state in cuprates has a $d$-wave superconducting energy gap, with nodes along the diagonals of the Brillouin zone 13]. A linearization of the Bogolyubov spectrum of quasiparticles around four nodes on the Fermi surface leads to another realization of gapless fermion excitations. Although from physical point of view the OAF state seems to be more complicated than the $d$-wave superconducting state, because the former is characterized by nonzero local currents violating time-reversal and translational symmetries, the Dirac description of OAF state turns out to be simpler since an external electromagnetic field enters into the theory in the same way as in $\mathrm{QED}_{2+1}$.

Carbon-based materials, e.g. pyrolytic graphite and carbon nanotubes present one more example of the planar systems with the relativistic dispersion law 14, 15, 16. From an experimental point of view these materials are probably the most promising because the quality of available samples already allows one to observe SdH effect [17, 18] and to study quantum effects that are due to a high magnetic field [19] (see Ref. 20] for a review).

The relativistic theories in an external magnetic field have been the subject of research in quantum field theory for many years (for reviews see Ref. 21]). The extraction of MO from the general expressions presents a rather subtle and interesting problem that was investigated in Refs. 22, 23, 24, 25, 26]. However these studies of the MO in QED are mostly concentrated on the field theoretical aspects.

The purpose of the present paper is to make a systematic study of the MO in $\mathrm{QED}_{2+1}$ devoting special attention to the link with planar condensed matter systems and to quantities that are particularly important for condensed matter theory, e.g. density of states (DOS), thermodynamic potential and magnetization. To make the theory more realistic we also include into the model the effect of scattering from impurities, by considering Landau levels with field and temperature independent width.

We begin by presenting in Sec. III three 2D models mentioned above that can be studied using the same effective relativistic Lagrangian written in Sec. IID in the presence of an external magnetic field. The Green's function necessary for subsequent calculations is introduced in Sec. III and the DOS oscillations are studied in Sec. IV both with and without scattering from impurities. The general representation for the thermodynamic potential in terms of the DOS and its link to the corresponding nonrelativistic potential are considered in Sec. V] In Sec. VI we derive the expression for the thermodynamic potential at $T=0$ in the absence of impurities with explicitly extracted MO (the calculational details and an alternative representation for the thermodynamic potential in terms of the generalized zeta function are given in Appendices $\mathrm{A}$ and $\mathrm{B}$. In Sec. VIA and at the end of Sec. VIIIC we discuss a possibility of detecting a gap that may open in the spectrum of one of the systems discussed above. The analytical expression for the magnetization at $T=0$ in the absence of impurities written in terms of Bernoulli polynomials is given in Sec. VIII In Sec. VIII] we generalize the results obtained in the previous sections to the presence of impurities (Secs. VIIIA and VIIIB with the calculational details presented in Appendix Cl) and in Sec. VIIIC for nonzero temperature. In Conclusions, Sec. [X] we give a concise summary of the obtained results.

\section{MODEL}

There are many planar condensed matter models that in the low energy sector can be reduced to $\mathrm{QED}_{2+1}$ form and here we briefly describe some of them underlining the main assumptions leading to the Dirac-like form of the effective Hamiltonians. 


\section{A. Hamiltonian of $d$-density wave state}

Due to $d_{x^{2}-y^{2}}$ momentum dependence of the gap in the OAF states it is also called $d$-density wave (DDW) state [27]. The mean-field phenomenological Hamiltonian describing this state can be written as [12, 28]

$$
H^{\mathrm{DDW}}=\sum_{\sigma=\uparrow, \downarrow} \int_{\mathrm{RBZ}} \frac{d^{2} k}{(2 \pi)^{2}} \chi_{\sigma}^{\dagger}(\mathbf{k})\left[\varepsilon(\mathbf{k}) \sigma_{3}-\mu \hat{I}-D(\mathbf{k}) \sigma_{2}\right] \chi_{\sigma}(\mathbf{k}),
$$

where the spinors

$$
\chi_{\sigma}(\mathbf{k})=\left(\begin{array}{c}
c_{\sigma}(\mathbf{k}) \\
c_{\sigma}(\mathbf{k}+\mathbf{Q})
\end{array}\right), \quad \chi_{\sigma}^{\dagger}(\mathbf{k})=\left(\begin{array}{ll}
c_{\sigma}^{\dagger}(\mathbf{k}) & c_{\sigma}^{\dagger}(\mathbf{k}+\mathbf{Q})
\end{array}\right),
$$

are composed from creation and annihilation operators $c_{\sigma}^{\dagger}(\mathbf{k}), c_{\sigma}(\mathbf{k})$ for momentum $\mathbf{k}$ and spin $\sigma$, the single particle energy is $\varepsilon(\mathbf{k})=-2 t\left(\cos k_{x} a+\cos k_{y} a\right)$ with $t$ being the hopping parameter, $\mu$ is the chemical potential, $D(\mathbf{k})=$ $\frac{D_{0}}{2}\left(\cos k_{x} a-\cos k_{y} a\right)$ is the $d$-density wave gap and $\mathbf{Q}=(\pi / a, \pi / a)$ is the wave vector at which the density-wave ordering takes place. The integral is over the reduced Brillouin zone RBZ and $\sigma_{i}$ are Pauli matrices. Throughout the paper $\hbar=c=k_{B}=1$ units are chosen, unless stated explicitly otherwise.

This Hamiltonian describes the excitations with the spectrum $E(\mathbf{k})=-\mu \pm \sqrt{\varepsilon^{2}(\mathbf{k})+D^{2}(\mathbf{k})}$. Linearizing the spectrum about the four nodes $\mathbf{N}_{i}=( \pm \pi / 2 a, \pm \pi / 2 a)$ with $i=1, \ldots, 4$ at half-filling $(\mu=0)$ one obtains $E(\mathbf{k})=$ $-\mu \pm \sqrt{v_{F}^{2} k_{x}^{2}+v_{D}^{2} k_{y}^{2}}$, where the Fermi velocity calculated for half-filling $v_{F}=|\partial \varepsilon(\mathbf{k}) / \partial \mathbf{k}|_{\mathbf{k}=\mathbf{N}} \mid=2 \sqrt{2} t a$, the DDW gap velocity $v_{D}=|\partial D(\mathbf{k}) / \partial \mathbf{k}|_{\mathbf{k}=\mathbf{N}} \mid=\frac{1}{\sqrt{2}} D_{0} a$, and the momenta $k_{x}$ and $k_{y}$ are given in the local nodal coordinate system (see Fig. 2 of Ref. [28]).

Using this linearized spectrum and inserting the vector potential in a way that preserves the charge conservation for the original Hamiltonian (2.1), one can arrive 29] at the following Dirac Lagrangian describing the quasiparticles in the vicinity of one of the nodes, e.g. $\mathbf{N}_{i=1}=(\pi / 2 a, \pi / 2 a)$

$$
\mathcal{L}=\bar{\chi}_{\sigma}^{i}(x) i \tilde{\gamma}^{\nu} D_{\nu} \chi_{\sigma}^{i}(x), \quad x=(t, \mathbf{r})
$$

where $\bar{\chi}_{\sigma}^{i}(x)=\chi_{\sigma}^{i \dagger}(x) \sigma_{1}$ is the Dirac conjugated spinor, $i$ labels the node $\mathbf{N}_{i}$, the covariant derivatives $D_{\nu}$ are

$$
D_{\nu}= \begin{cases}\hbar \partial_{t}-i e A_{0}(x), & \nu=0 \\ v_{F}\left(\hbar \partial_{x}-i \frac{e}{c} A_{1}(x)\right), & \nu=1 \\ v_{\Delta}\left(\hbar \partial_{y}-i \frac{e}{c} A_{2}(x)\right), & \nu=2\end{cases}
$$

and the $\gamma$ matrices are

$$
\tilde{\gamma}^{\nu}=\left(\sigma_{1},-i \sigma_{2}, i \sigma_{3}\right), \quad\left\{\tilde{\gamma}^{\mu}, \tilde{\gamma}^{\nu}\right\}=2 \hat{I}_{2} g^{\mu \nu}, \quad g^{\mu \nu}=\operatorname{diag}(1,-1,-1), \quad \mu, \nu=0,1,2 .
$$

The chemical potential $\mu$ can be introduced in the Lagrangian (2.3) by choosing $A_{0}=\mu / e$ and the sum over the spin components in Eq. (2.3) can be regarded as an additional flavor index.

In $2+1$ dimensions, there are two inequivalent representations of Dirac algebra (see e.g. [30]):

$$
\begin{array}{ll}
\hat{\gamma}^{0}=\sigma_{3}, & \hat{\gamma}^{1}=i \sigma_{1}, \quad \hat{\gamma}^{2}=i \sigma_{2}, \\
\hat{\gamma}^{0}=-\sigma_{3}, & \hat{\gamma}^{1}=-i \sigma_{1}, \quad \hat{\gamma}^{2}=-i \sigma_{2},
\end{array}
$$

which correspond to right and left handed coordinate systems. As one can check, our $\gamma$-matrices (2.5) correspond to the representation (2.6b) if one makes a unitary transformation $\hat{\chi}^{1}=U \chi^{1}, \hat{\gamma}^{\mu}=U \tilde{\gamma}^{\mu} U^{-1}$ with $U=(1 / 2)\left(\hat{I}_{2}-i \sigma_{1}-\right.$ $i \sigma_{2}+i \sigma_{3}$ ) Since the physical properties of the system depend only on the algebra that these matrices obey, one can directly work with the more commonly used representation (2.6) instead of (2.5).

Dealing with (2.3) one should not forget that all physical quantities involve the summation over the 4 nodes present in the original Hamiltonian (2.1). In fact, only two neighboring nodes, e.g. $\mathbf{N}_{i=1}=(\pi / 2 a, \pi / 2 a)$ and $\mathbf{N}_{i=2}=(\pi / 2 a,-\pi / 2 a)$ are nonequivalent in RBZ and the corresponding local nodal coordinate systems are related to each other by parity transformation $\left(k_{x}, k_{y}\right) \rightarrow\left(k_{x},-k_{y}\right)$ with the simultaneous interchange $v_{F} \leftrightarrow v_{D}$. Thus the sum over nonequivalent nodes can be taken into account by doubling spinors

$$
\Upsilon_{\sigma}=\left(\begin{array}{l}
\psi_{\sigma}^{1} \\
\psi_{\sigma}^{2}
\end{array}\right)
$$


and using the reducible representation of $\gamma$-matrices, $\gamma^{\mu}=\left(\sigma_{3}, i \sigma_{1}, i \sigma_{2}\right) \otimes \sigma_{3}$ :

$$
\gamma^{0}=\left(\begin{array}{cc}
\sigma_{3} & 0 \\
0 & -\sigma_{3}
\end{array}\right), \quad \gamma^{1}=\left(\begin{array}{cc}
i \sigma_{1} & 0 \\
0 & -i \sigma_{1}
\end{array}\right), \quad \gamma^{2}=\left(\begin{array}{cc}
i \sigma_{2} & 0 \\
0 & -i \sigma_{2}
\end{array}\right)
$$

with the following Lagrangian density

$$
\mathcal{L}=\bar{\Upsilon}_{\sigma}(x) i \gamma^{\nu} D_{\nu} \Upsilon_{\sigma}(x)
$$

\section{B. Hamiltonian of $d$-wave superconducting state}

In contrast to the hypothetical DDW state, $d$-wave superconductivity $(d \mathrm{SC})$ is observed in cuprate superconductors 13]. It can be described by the Bogolyubov - de Gennes Hamiltonian

$$
\begin{aligned}
H_{\mathrm{BdG}} & =\int d^{2} r \Psi^{\dagger}(t, \mathbf{r}) \tau_{3}\left[\varepsilon\left(-i \nabla-\tau_{3} \frac{e}{c} \mathbf{A}(\mathbf{r})\right)-\mu\right] \Psi(t, \mathbf{r}) \\
& -\int d^{2} r_{1} \int d^{2} r_{2}\left[\Delta^{\dagger}\left(t, \mathbf{r}_{1} ; \mathbf{r}_{2}\right) \Psi^{\dagger}\left(t, \mathbf{r}_{1}\right) \tau_{-} \Psi\left(t, \mathbf{r}_{2}\right)+\Psi^{\dagger}\left(t, \mathbf{r}_{1}\right) \tau_{+} \Psi\left(t, \mathbf{r}_{2}\right) \Delta\left(t, \mathbf{r}_{1} ; \mathbf{r}_{2}\right)\right],
\end{aligned}
$$

where $\Psi$ and $\Psi^{\dagger}=\left(\psi_{\uparrow}^{\dagger}, \psi_{\downarrow}\right)$ are the standard Nambu spinors, $\varepsilon(\mathbf{p})$ is the single particle energy, $\tau_{ \pm}=\left(\tau_{1} \pm i \tau_{2}\right) / 2$ and $\Delta\left(t, \mathbf{r}_{1} ; \mathbf{r}_{2}\right)$ is the bilocal gap operator the Hermitian conjugate of which includes the transpose in the functional sense, i.e. $\Delta^{\dagger}\left(t, \mathbf{r}_{1} ; \mathbf{r}_{2}\right) \equiv\left[\Delta\left(t, \mathbf{r}_{2} ; \mathbf{r}_{1}\right)\right]^{*}$. The nontrivial dependence of $\Delta\left(t, \mathbf{r}_{1} ; \mathbf{r}_{2}\right)$ on the relative coordinate $\mathbf{r}_{1}-\mathbf{r}_{2}$ is supposed to describe $d$-wave pairing state. Further simplification of the pairing term of Eq. (2.10) is possible if one assumes that the amplitude of the pairing term is a constant with respect to $t$ and the center of mass coordinate $\mathbf{R}=\left(\mathbf{r}_{1}+\mathbf{r}_{2}\right) / 2$. For cuprates this assumption may well be justified even above the critical temperature, $T_{c}$. Below $T_{c}$ in the vortex state this assumption corresponds to neglecting vortex core contributions and considering pure "phase vortices". Since we deal with the amplitude of the bilocal complex field $\Delta\left(t, \mathbf{r}_{1}, \mathbf{r}_{2}\right)$, it is natural to consider also its phase $\theta(t, \mathbf{R})$. There are, however, some subtleties in writing the continuum version of the pairing term of (2.10) in an explicitly gauge invariant form that can be solved by choosing an appropriate form of the differential operator for $\hat{\Delta}$ (see Refs. 31, 32]). Since we look for the low-energy quasiparticle excitations near four gap nodes, we write the linearized Hamiltonian for one of these nodes as (see e.g. [28, 31])

$$
H_{d S C}=\Psi^{\dagger}(x)\left[i v_{F} \tau_{3}\left(\partial_{x}-i \frac{e}{c} \tau_{3} A_{x}\right)+i v_{\Delta} \tau_{+} e^{i \theta(x) / 2} \partial_{y} e^{i \theta(x) / 2}+i v_{\Delta} \tau_{-} e^{-i \theta(x) / 2} \partial_{y} e^{-i \theta(x) / 2}\right] \Psi(x),
$$

where $v_{F}$ is the Fermi velocity, $v_{\Delta}=|\partial \Delta(\mathbf{k}) / \partial \mathbf{k}|_{\mathbf{k}=\mathbf{N}} \mid$ is the gap velocity defined by the slope of the superconducting gap $\Delta(\mathbf{k})$. Both velocities are calculated in the nodal points on the true Fermi surface of the system, but not at half-filling as for the DDW case. The linearization puts restrictions on the domain of validity of the Hamiltonian (2.11) which, however, remains rather wide [33].

Finally, introducing the Dirac conjugated spinor $\bar{\eta}^{i}=\Psi^{i \dagger} \tau_{2}$ we can obtain from the Hamiltonian (2.11) a Lagrangian similar to Eq. (2.3), but with the vector potential that couples only with the $v_{F}$ term. Again making, if necessary, a unitary transformation, one can combine spinors $\eta^{i}$ originating from two opposite nodes into one four-component spinor.

Thus in the absence of the field the low lying quasiparticle excitations in the $d \mathrm{SC}$ are described by the relativistic dispersion law

$$
E(\mathbf{k})= \pm \sqrt{v_{F}^{2} k_{x}^{2}+v_{\Delta}^{2} k_{y}^{2}}
$$

where the momenta $k_{x}$ and $k_{u}$ are given in the local nodal coordinate system. Experimental values of $v_{F}$ and $v_{\Delta}$ for cuprates can be found in Ref. [34], for example, the value of $v_{F} \sim 2.5 \times 10^{5} \mathrm{~m} / \mathrm{s}$ and the anisotropy of the Dirac cone $v_{F} / v_{\Delta}$ varies between 10 and 20 depending on doping and compound.

There are two important differences between DDW and $d \mathrm{SC}$ Hamiltonians. Making a gauge transformation that removes the phase from the last term of Eq. (2.11), we observe that quasiparticles in superconductor do not couple simply to the vector potential $\mathbf{A}$ corresponding to the magnetic field $\mathbf{B}=\nabla \times \mathbf{A}$, but to the supercurrent $\nabla \theta-(2 e / \hbar c) \mathbf{A}$, where $\theta$ is the phase of the superconducting order parameter. This difference between DDW and $d$ SC cases leads to a conclusion that in an external magnetic field the nodal quasiparticles of DDW state form Landau levels [35], while in $d$ SC state Landau levels are strongly mixed 36. Nevertheless we presented $d$ SC Hamiltonian (2.11) here due to its practical importance and the fact that the simplified physical picture based on the Landau levels [37, 38] may become 
relevant for higher energies [39] and can be regarded as the first approximation to a more complicated case of the vortex state in $d$-wave superconductors.

The second difference comes from the fact that for DDW state chemical potential $\mu$ is explicitly present in the Lagrangian (2.3), while for $d \mathrm{SC}$ state it was absorbed in the definition of the Fermi velocity $v_{F}$ on the Fermi surface. The origin of this difference can be traced back to the different structures of the Hamiltonians (2.1) and (2.10). One can also say that in $d \mathrm{SC}$ state the chemical potential of nodal quasiparticles is zero, so that when the applied field is changed the corresponding Landau levels (even if they were formed) cannot cross the chemical potential and produce dHvA effect [37].

\section{Layered graphite}

The semimetallic energy band structure of a single graphene sheet has the conduction and valence $\pi$ bands with the energy dispersion [40]

$$
E\left(k_{x}, k_{y}\right)= \pm t \sqrt{1+4 \cos \frac{\sqrt{3} k_{x} a}{2} \cos \frac{k_{y} a}{2}+4 \cos ^{2} \frac{k_{y} a}{2}},
$$

where $t \approx 3 e V, a=\sqrt{3} a_{C C}=2.46 \AA$ is the lattice constant of two dimensional graphite, $a_{C C}$ is the distance between two nearest carbon atoms. These two bands touch each other and cross the Fermi level in six K points located at the corners of the hexagonal 2D Brillouin zone, but only two of them, for example, $\mathbf{K}=(2 \pi / a)(1 / \sqrt{3}, 1 / 3)$ and $\mathbf{K}^{\prime}=(2 \pi / a)(0,2 / 3)$ are inequivalent due to the periodicity of the Brillouin zone. The low-energy excitations can be studied by taking the continuum limit $a \rightarrow 0$ at any two independent $\mathrm{K}$ points labelled as $j=1,2$. They have a linear dispersion $E_{k}= \pm v_{F} k$ with $v_{F}=(\sqrt{3} / 2) t a \approx 9.7 \times 10^{5} \mathrm{~m} / \mathrm{s}$. These excitations can be formally described by a pair of two-component (Weyl) spinors $\psi_{j \sigma}$, which are composed of the Bloch states residing on the two different sublattices of the bi-particle hexagonal lattice of the graphene sheet. The Hamiltonian describing these excitations, for example, in the point $\mathbf{K}$ coincides with the free Dirac one [14, 16]

$$
H=\sum_{\sigma=\uparrow, \downarrow} \int \frac{d^{2} k}{(2 \pi)^{2}} \bar{\psi}_{1 \sigma}(t, \mathbf{k})\left(\bar{\gamma}^{1} k_{x}+\bar{\gamma}^{2} k_{y}\right) \psi_{1 \sigma}(t, \mathbf{k}),
$$

where the momentum $\mathbf{k}=\left(k_{x}, k_{y}\right)$ is already given in a local coordinate system associated with a chosen $\mathrm{K}$ point, $\bar{\psi}_{1 \sigma}=\psi_{1 \sigma}^{\dagger} \bar{\gamma}^{0}$ and $\bar{\gamma}^{0,1,2}=\left(\sigma_{3}, i \sigma_{2},-i \sigma_{1}\right)$. This representation of $\bar{\gamma}$-matrices can be mapped in the representation (2.6a) by using a unitary transformation with $U=(1 / \sqrt{2})\left(\hat{I}+i \sigma_{3}\right)$. Since the local coordinate system for the point $\mathbf{K}^{\prime}$ is related to the system associated with the point $\mathbf{K}$ by a parity transformation, these two spinors can be again combined in one four-component Dirac spinor $\Psi_{\sigma}=\left(\psi_{1 \sigma}, \psi_{2 \sigma}\right)$. The number of spin components $N$ has to be regarded as an adjustable parameter and $N=2$ corresponds to the physical case. Finally, the Lagrangian density of noninteracting quasiparticles reads

$$
\mathcal{L}_{0}=\sum_{\sigma=1}^{N} v_{F} \bar{\Psi}_{\sigma}(t, \mathbf{r})\left(\frac{i \gamma^{0}\left(\partial_{t}+i \mu\right)}{v_{F}}-i \gamma^{1} \partial_{x}-i \gamma^{2} \partial_{y}\right) \Psi_{\sigma}(t, \mathbf{r}),
$$

where $\bar{\Psi}_{\sigma}=\Psi_{\sigma}^{\dagger} \gamma^{0}$ and $4 \times 4 \gamma$-matrices are either $\left(\sigma_{3}, i \sigma_{3},-i \sigma_{1}\right) \otimes \sigma_{3}[41]$ or can be taken from the unitary equivalent representation (2.8) 14, 42]. Since the terms with $\partial_{x, y}$ in Eq. 2.15) originate from the usual kinetic term of the tight-binding Hamiltonian, vector potential $\mathbf{A}$ can be inserted in the Lagrangian (2.15) using a minimal coupling prescription. Finally we note that three-dimensional version of the Dirac Hamiltonian (2.14) was used in Ref. [9] to describe an unusual magnetoresistance in the two doped chalcogenides $\mathrm{Ag}_{2+\delta}$ Se and $\mathrm{Ag}_{2+\delta} \mathrm{Te}$.

\section{Model relativistic Lagrangian}

As we have seen, many models of planar condensed matter systems result in the Dirac type form of the effective low energy theory. Thus as a starting point of the present paper we choose the following Lagrangian

$$
\mathcal{L}=\bar{\psi}_{i}\left(i \gamma^{\mu} D_{\mu}-\Delta\right) \psi_{i}, \quad \mu=0,1,2,
$$


where $D_{\mu}=\partial_{\mu}-i e A_{\mu}$ is the covariant derivative, $\bar{\psi}_{i} \equiv \psi_{i}^{\dagger} \gamma^{0}$ is the Dirac conjugated spinor and the vector potential for the external magnetic field $\mathbf{B}$ perpendicular to the plane is taken in the symmetric gauge

$$
\mathbf{A}=\left(-\frac{B}{2} x_{2}, \frac{B}{2} x_{1}\right) .
$$

The nonzero chemical potential $\mu$ will also be taken into account by choosing $A_{0}=\mu / e$, in the energy-momentum space this corresponds to a shifting $\omega \rightarrow \omega+\mu$. We assume that the fermions carry an additional flavor index $i=1, \ldots, N$ which can be used to calculate the sum over equivalent nodes in the case of $d \mathrm{SC}$ case and to sum over the spin components in the cases of DDW and graphite. In (2.16) we have already set the velocities $c=v_{F}=v_{D}=v_{\Delta}=1$, so that we consider the "isotropic Dirac cone". When it is needed they can be restored according to the prescription discussed in Refs. [29, 43]. The Dirac $\gamma$-matrices are taken in the reducible four-component representation and they obey the Clifford (Dirac) algebra, $\gamma^{\mu} \gamma^{\nu}+\gamma^{\nu} \gamma^{\mu}=2 \hat{I}_{4} g^{\mu \nu}$. The explicit form of the $\gamma$-matrices is given in Eq. (2.8) and the symmetry properties of the Lagrangian with reducible four-dimensional representation are discussed, for example, in [30, 42, 44]. We have also included a mass (gap) term $\Delta$ in the Lagrangian (2.16). The physical origin of this gap depends on the underlying system we consider. For example, it is well known that an external magnetic field is a strong catalyst in generating such a gap for Dirac fermions (the phenomenon of magnetic catalysis) [4]. Usually the opening of the gap marks an important transition which occurs in the system. In particular, in the case of pyrolytic graphite a poor screening of the Coulomb interaction may lead to excitonic instability resulting in the opening of the gap in the electronic spectrum and manifesting itself through the onset of an insulating charge density wave (see e.g. [41, 42]). There are, however, many obstacles in experimental detection of such a gap, so that in the context of the MO studies we consider in Secs. VIA and VIIIC a possibility for its detection using dHvA and SdH effects.

A full theory of MO should also include the Zeeman interaction term which leads to a spin factor in the LK formula. Here, however, we neglect this term motivated by the fact that for the relativistic spectrum the MO may become observable for the relatively low fields when the spin splitting is still small. If necessary, the Zeeman term can be added explicitly both to the original Hamiltonian (2.1) and the Lagrangian (2.16).

\section{SPECTRAL FUNCTION OF DIRAC QUASIPARTICLES IN AN EXTERNAL FIELD}

The Green's function of Dirac fermions described by the Lagrangian (2.16) in an external field given by the vector potential (2.17) reads

$$
S(x-y)=\exp \left(i e \int_{y}^{x} A_{\lambda} d z^{\lambda}\right) \tilde{S}(x-y)
$$

where $\tilde{S}$ is the translation invariant part $\tilde{S}$ of the Green's function. Its derivation using the Schwinger proper-time method and decomposition over Landau level poles has been discussed in many papers (see e.g. Refs. 29, 44, 45]), so that here we begin with the spectral function associated with the translationary invariant part $\tilde{S}$ of the Green's function

$$
A(\omega, \mathbf{p})=\frac{1}{2 \pi i}\left[\tilde{S}^{A}(\omega-i 0, \mathbf{p})-\tilde{S}^{R}(\omega+i 0, \mathbf{p})\right]
$$

where the retarded, $\tilde{S}^{R}$, and advanced, $\tilde{S}^{A}$, Greens' functions are written in the energy-momentum representation. This spectral function decomposed over Landau levels reads [43]

$$
A(\omega, \mathbf{p})=\exp \left(-\frac{\mathbf{p}^{2}}{|e B|}\right) \sum_{n=0}^{\infty}(-1)^{n}\left[\frac{\left(\gamma^{0} M_{n}+\Delta\right) f_{1}(\mathbf{p})+f_{2}(\mathbf{p})}{2 M_{n}} \delta\left(\omega-M_{n}\right)+\frac{\left(\gamma^{0} M_{n}-\Delta\right) f_{1}(\mathbf{p})-f_{2}(\mathbf{p})}{2 M_{n}} \delta\left(\omega+M_{n}\right)\right],
$$

where $M_{n}=\sqrt{\Delta^{2}+2 e B n}$ and

$$
f_{1}(\mathbf{p})=2\left[P_{-} L_{n}\left(2 \frac{\mathbf{p}^{2}}{|e B|}\right)-P_{+} L_{n-1}\left(2 \frac{\mathbf{p}^{2}}{|e B|}\right)\right], \quad f_{2}(\mathbf{p})=4\left(p_{1} \gamma^{1}+p_{2} \gamma^{2}\right) L_{n-1}^{1}\left(2 \frac{\mathbf{p}^{2}}{|e B|}\right),
$$

with $P_{ \pm}=\left(1 \pm \operatorname{sgn}(e B) i \gamma^{1} \gamma^{2}\right) / 2$ being projectors and $L_{n}, L_{n}^{1}$ Laguerre's polynomials $\left(L_{-1}^{1} \equiv 0\right)$. In what follows, for convenience, we take $e B>0$. The chemical potential $\mu$, as mentioned above, has to be taken into account via the shift $\omega \rightarrow \omega+\mu$. 
In contrast to the nonrelativistic Landau levels with the energies $E_{n}=\frac{e \hbar B}{m c}\left(n+\frac{1}{2}\right)$ (here $m$ is the effective mass of the carriers), in the relativistic problem with zero gap $\Delta$ the energies are $E_{n}=\sqrt{\frac{e \hbar v_{F} v_{2}}{c} B 2 n}, n=0,1, \ldots$, where we restored all parameters $\left(v_{2} \equiv v_{F}, v_{D}\right.$ or $v_{\Delta}$ depending on the model we consider) to show explicitly the differences. For the nonrelativistic problem the distance between Landau levels coincides with the cyclotron frequency, $\omega_{c}=e \hbar B /(m c)$, so that $\omega_{c}[\mathrm{~K}] \sim 1.35 B$ [Tesla $] m_{e} / m$, where $m_{e}$ is the electron mass, while for the relativistic problem the corresponding energy scale, characterizing the distance between Landau levels, is

$$
\omega_{L}=\sqrt{\frac{\hbar v_{F} v_{2} 2 e B}{c}}[\mathrm{~K}]=4.206 \times 10^{-4} \sqrt{\frac{v_{2}}{v_{F}}} v_{F}[\mathrm{~m} / \mathrm{s}] \sqrt{B[\text { Tesla }]},
$$

where $v_{F}$ is given in $\mathrm{m} / \mathrm{s}$. For example, choosing $m_{e} / m=1$ we estimate that $\omega_{c} \sim 1 \mathrm{~K} \cdot B$ [Tesla], but this value can be increased by using metals such as Bi with a large ratio $m_{e} / \mathrm{m}$. Making the estimate for the relativistic case with a rather small $v_{F}=2 \times 10^{5} \mathrm{~m} / \mathrm{s}$ and $v_{F} / v_{2}=20$, which are typical values of the parameters for the DDW model, we obtain $\omega_{L} \sim 18 K \cdot \sqrt{B[\text { Tesla }]}$ which shows that in the systems with the linear dispersion law the quantum condition favorable for MO persists to rather high temperatures and small fields [9]. Moreover, repeating the estimate for the Fermi velocity in graphite, $v_{F}=9.7 \times 10^{5} \mathrm{~m} / \mathrm{s}$, we obtain even larger $\omega_{L} \sim 400 K \cdot \sqrt{B[\text { Tesla }]}$. We note that since the Zeeman term has the same magnitude as $\omega_{c}$, it is indeed small comparing to $\omega_{L}$ and can be safely neglected.

In order to consider the MO for a more realistic case, one should introduce the effect of quasiparticle scattering that results in a Dingle factor in the expression for the amplitude of MO. In general, this can be done by considering dressed fermion propagators that include the self-energy $\Sigma(\omega)$ due to the scattering from impurities. Up to now the problem of scattering from impurities in the presence of a magnetic field does not have yet a satisfactory solution. Therefore, here we choose the case of constant width $\Gamma=\Gamma(\omega=0)=-\operatorname{Im} \Sigma^{R}(\omega=0)=1 /(2 \tau), \tau$ being a mean free time of quasiparticles, so that the $\delta$-like quasiparticle peaks corresponding to the Landau levels in Eq. (3.2) acquire a Lorentzian shape:

$$
\delta\left(\omega \pm M_{n}\right) \rightarrow \frac{1}{\pi} \frac{\Gamma}{\left(\omega \pm M_{n}\right)^{2}+\Gamma^{2}}
$$

Such a broadening of Landau levels with a constant $\Gamma$ was found to be a rather good approximation valid in not very strong magnetic fields [1, 46]. Definitely, the treatment of disorder in the presence of the magnetic field in such a simplified manner should be considered as only the first step until further progress in this problem is achieved (in connection with this, see, Ref. [47]). The technical advantage of the approximation $\Gamma=$ const is that one can firstly consider dHvA oscillations with $\delta$-like Landau levels at $T=0$ and only afterwards introduce the effect of level broadening due to the finite temperature and quasiparticle scattering convoluting the final results with the appropriate distribution functions (see, Refs. 1, 48] and Sec. (V). However, this simplification is only valid if all Landau levels have the same width.

\section{OSCILLATIONS OF DENSITY OF STATES}

We begin with the calculation of the quasiparticle density of states (DOS) $D_{0}(\epsilon)$ in the absence of scattering $(\Gamma=0)$, which is expressed in terms of the spectral function (3.3) as

$$
D_{0}(\epsilon)=\int_{\mathrm{B}} \frac{d^{2} p}{(2 \pi)^{2}} \operatorname{tr}\left[\gamma_{0} A(\epsilon, \mathbf{p})\right]
$$

where the domain of integration $B$ is chosen to preserve the volume of the original Brillouin zone. We also assume that the summation over spin states that are identical in the absence of Zeeman term is included in the definition of trace in $D(\epsilon)$. This, as mentioned above, can be done by taking an appropriate value of $N$.

Evaluating the trace and expanding the limits of integration over momenta to $\infty$, we get the DOS as the sum of delta functions of Landau's level energies:

$$
D_{0}(\epsilon)=\frac{N e B}{2 \pi}\left[\delta(\epsilon-\Delta)+\delta(\epsilon+\Delta)+2 \sum_{n=1}^{\infty}\left(\delta\left(\epsilon-M_{n}\right)+\delta\left(\epsilon+M_{n}\right)\right)\right] .
$$

The fact that the original Brillouin zone has a finite volume can be taken into account later using the finite limits of integration if necessary and/or by implying that the $\operatorname{DOS} D(\epsilon)$ is multiplied by the factor $\theta(\Lambda-|\epsilon|)$, where $\Lambda$ is the bandwidth. 
The broadening of Landau levels due to the scattering is taken into account according to the prescription given by Eq. (3.6). It is easy to see that this prescription corresponds to the convolution of the density of states $D_{0}(\epsilon)$ with the Lorentz distribution $P_{\Gamma}(\omega)=\Gamma /\left[\pi\left(\omega^{2}+\Gamma^{2}\right)\right]$

$$
D(\epsilon)=\int_{-\infty}^{\infty} d \omega P_{\Gamma}(\omega-\epsilon) D_{0}(\omega), \quad \int_{-\infty}^{\infty} d \omega P_{\Gamma}(\omega)=1
$$

In fact, instead of the Lorentz distribution any other normalized probability distribution can be used [1] in order to introduce phenomenologically the energy level width. In what follows we shall use the Lorentz distribution which allows analytical calculations. Physically, the Lorentz distribution corresponds to an extremely strong disorder since all moments of this distribution diverge. The quasiparticle DOS at the Fermi surface can be obtained by evaluating Eq. (4.3) for $\epsilon=\mu$. Eq. (4.2) can be also rewritten as

$$
\begin{aligned}
D_{0}(\epsilon) & =\frac{N e B|\epsilon|}{\pi}\left[\delta\left(\epsilon^{2}-\Delta^{2}\right)+2 \sum_{n=1}^{\infty} \delta\left(\epsilon^{2}-\Delta^{2}-2 e B n\right)\right] \\
& =\frac{N e B}{2 \pi} \operatorname{sgn}(\epsilon) \frac{d}{d \epsilon}\left[\theta\left(\epsilon^{2}-\Delta^{2}\right)+2 \sum_{n=1}^{\infty} \theta\left(\epsilon^{2}-\Delta^{2}-2 e B n\right)\right],
\end{aligned}
$$

where $\theta$ is the step function. Note that $N e B /(2 \pi)$ is the density of the Landau levels. Using the Poisson summation formula

$$
\frac{1}{2} F(0)+\sum_{n=1}^{\infty} F(n)=\int_{0}^{\infty} F(x) d x+2 \operatorname{Re} \sum_{k=1}^{\infty} \int_{0}^{\infty} F(x) e^{2 \pi i k x} d x
$$

we find the sum over the Landau levels

$$
\sum_{n=1}^{\infty} \theta\left(\epsilon^{2}-\Delta^{2}-2 e B n\right)=\theta\left(\epsilon^{2}-\Delta^{2}\right)\left[-\frac{1}{2}+\frac{\epsilon^{2}-\Delta^{2}}{2 e B}+\sum_{k=1}^{\infty} \frac{\sin \left(\pi k \frac{\epsilon^{2}-\Delta^{2}}{e B}\right)}{\pi k}\right]
$$

Substituting now the last expression in Eq.4.4 we obtain the final expression for the DOS with zero broadening that can be written in three equivalent forms:

$$
\begin{aligned}
D_{0}(\epsilon) & =\frac{N}{2 \pi} \operatorname{sgn}(\epsilon) \frac{d}{d \epsilon}\left\{\theta\left(\epsilon^{2}-\Delta^{2}\right)\left[\epsilon^{2}-\Delta^{2}+2 e B \sum_{k=1}^{\infty} \frac{1}{\pi k} \sin \left(\frac{\pi k\left(\epsilon^{2}-\Delta^{2}\right)}{e B}\right)\right]\right\} \\
& =\frac{N}{2 \pi} \operatorname{sgn}(\epsilon) \frac{d}{d \epsilon}\left\{\theta\left(\epsilon^{2}-\Delta^{2}\right)\left[\epsilon^{2}-\Delta^{2}+\frac{2 e B}{\pi} \tan ^{-1}\left(\cot \left(\frac{\pi\left(\epsilon^{2}-\Delta^{2}\right)}{2 e B}\right)\right)\right]\right\} \\
& =\frac{N}{2 \pi} \operatorname{sgn}(\epsilon) \frac{d}{d \epsilon}\left\{\theta\left(\epsilon^{2}-\Delta^{2}\right)\left[\epsilon^{2}-\Delta^{2}-2 e B B_{1}\left(\frac{\epsilon^{2}-\Delta^{2}}{2 e B}\right)\right]\right\} .
\end{aligned}
$$

The summation in Eq. (4.7b) was done using the formula

$$
\sum_{n=1}^{\infty} \frac{\sin (\pi n x)}{n}=\tan ^{-1}\left(\frac{\sin (\pi x)}{1-\cos (\pi x)}\right)
$$

and Eq. (4.7c) is written using the Bernoulli polynomials $B_{n}(x)$ periodically continued beyond the interval [0,1]:

$$
B_{n}(x)=-\frac{2 \cdot n !}{(2 \pi)^{n}} \sum_{k=1}^{\infty} \frac{1}{k^{n}} \cos \left(2 \pi k x-\frac{n \pi}{2}\right), \quad n \geq 2, \quad 0 \leq x \leq 1 ; \quad n=1, \quad 0<x<1 .
$$

In fact, all the Bernoulli polynomials we dealt with in this paper depend on the fractional part $\bmod [x]$ of their argument $x$, i.e., $B_{n}(\bmod [x])$ (here $\bmod [x]$ is a shorthand notation for $x$ modulo 1 , i.e., $\bmod [x]=x-[x]$ where $[x]$ is the largest integer satisfying $[x] \leq x)$. In what follows, for brevity we omit the sign $\bmod []$ and write simply $B_{n}(x)$. 


\section{A. DOS in the presence of scattering}

Now we turn to calculating DOS in presence of the impurity scattering rate $\Gamma$ using convolution (4.3). Since $D_{0}(\epsilon)$ is an even function of $\epsilon$, the expression for $D(\epsilon)$ can be written as follows

$$
\begin{aligned}
& D(\epsilon)=\frac{N}{\pi^{2}} \operatorname{Im}\left\{(\epsilon+i \Gamma)\left[\int_{0}^{\Lambda^{2} / e B} \frac{d x}{x+z}+\frac{2}{\pi} \int_{0}^{\infty} \frac{d x}{(x+z)^{2}} \tan ^{-1}\left(\cot \frac{\pi x}{2}\right)\right]\right\}, \\
& x=\frac{\omega^{2}-\Delta^{2}}{e B}, \quad z=\frac{\Delta^{2}-(\epsilon+i \Gamma)^{2}}{e B},
\end{aligned}
$$

where we used Eq. (4.7b) for $D_{0}(\epsilon)$, represented $P_{\Gamma}$ as $P_{\Gamma}(\epsilon \pm \omega)=-\operatorname{Im}(\epsilon \pm \omega+i \Gamma)^{-1}$ and integrated by parts to obtain the second term in square brackets in Eq. (4.10a). We also put an explicit cutoff $\Lambda$ associated with the bandwidth in the first integral describing non-oscillating part of DOS.

It is easy to calculate that

$$
\operatorname{Im}\left[(\epsilon+i \Gamma) \int_{0}^{\Lambda^{2} / e B} \frac{d x}{x+z}\right]=\Gamma \ln \frac{\Lambda^{2}}{\sqrt{\left(\epsilon^{2}-\Delta^{2}-\Gamma^{2}\right)^{2}+4 \epsilon^{2} \Gamma^{2}}}+\epsilon \tan ^{-1} \frac{2 \epsilon \Gamma}{\Delta^{2}+\Gamma^{2}-\epsilon^{2}} .
$$

The second integral in Eq. (4.10) denoted as $I$ can be calculated if we take into account that the function $\tan ^{-1}(\cot (\pi x / 2))$ is periodic with the period $x=2$ so that we can write

$$
\begin{aligned}
I & =\frac{2}{\pi} \int_{0}^{\infty} \frac{d x}{(x+z)^{2}} \tan ^{-1}\left(\cot \frac{\pi x}{2}\right)=\frac{2}{\pi} \sum_{k=0}^{\infty} \int_{2 k}^{2 k+2} \frac{d x}{(x+z)^{2}} \tan ^{-1}\left(\cot \frac{\pi x}{2}\right) \\
& =\frac{2}{\pi} \sum_{k=0}^{\infty} \int_{0}^{2} \frac{d x}{(x+2 n+z)^{2}} \tan ^{-1}\left(\cot \frac{\pi x}{2}\right)=\frac{1}{2} \int_{0}^{1} d x(1-2 x) \zeta(2, x+z / 2),
\end{aligned}
$$

where $\zeta(s, x)$ is the generalized zeta-function and we used also that $\tan ^{-1}(\cot (\pi x / 2))=(\pi / 2)(1-x)$ for $0<x<2$. The last integral is evaluated using the properties of $\zeta$-function

$$
\int_{0}^{1} d x \zeta(2, x+z)=\frac{1}{z}, \quad \int_{0}^{1} d x x \zeta(2, x+z)=\Psi(1+z)-\ln z,
$$

so that we finally get

$$
I=-\psi\left(\frac{z}{2}\right)+\ln \frac{z}{2}-\frac{1}{z} .
$$

Thus, we express the DOS in terms of the digamma function $\psi$ :

$$
D(\epsilon)=\frac{N}{\pi^{2}}\left\{\Gamma \ln \frac{\Lambda^{2}}{2 e B}-\operatorname{Im}\left[(\epsilon+i \Gamma)\left(\psi\left(\frac{\Delta^{2}-(\epsilon+i \Gamma)^{2}}{2 e B}\right)+\frac{e B}{\Delta^{2}-(\epsilon+i \Gamma)^{2}}\right)\right]\right\}
$$

or, in equivalent form

$$
D(\epsilon)=\frac{N}{\pi^{2}} \Gamma \ln \frac{\Lambda^{2}}{2 e B}+\frac{N e B}{\pi^{2}} \frac{d}{d \epsilon} \operatorname{Im}\left[\ln \Gamma\left(\frac{\Delta^{2}-(\epsilon+i \Gamma)^{2}}{2 e B}\right)+\frac{1}{2} \ln \left(\frac{\Delta^{2}-(\epsilon+i \Gamma)^{2}}{2 e B}\right)\right] .
$$

It is evident that the DOS oscillations are contained in the $\psi$-function when the real part of its argument becomes negative. They can be extracted in explicit form using the relationship

$$
\psi(-z)=\psi(z)+\frac{1}{z}+\pi \cot (\pi z)
$$


Hence we get

$$
\begin{aligned}
& \psi\left(\frac{\Delta^{2}-(\epsilon+i \Gamma)^{2}}{2 e B}\right)=\psi\left(\frac{\Delta^{2}-(\epsilon+i \Gamma)^{2}}{2 e B}\right)\left(\theta\left(\epsilon^{2}-\Delta^{2}-\Gamma^{2}\right)+\theta\left(\Delta^{2}+\Gamma^{2}-\epsilon^{2}\right)\right) \\
& =\operatorname{Re} \psi\left(\frac{\left|\epsilon^{2}-\Delta^{2}-\Gamma^{2}\right|-2 i \epsilon \Gamma}{2 e B}\right)-i \operatorname{sgn}\left(\epsilon^{2}-\Delta^{2}-\Gamma^{2}\right) \operatorname{Im} \psi\left(\frac{\left|\epsilon^{2}-\Delta^{2}-\Gamma^{2}\right|-2 i \epsilon \Gamma}{2 e B}\right) \\
& +\theta\left(\epsilon^{2}-\Delta^{2}-\Gamma^{2}\right)\left[\frac{2 e B}{\epsilon^{2}-\Delta^{2}-\Gamma^{2}+2 i \epsilon \Gamma}+\pi \cot \pi \frac{\epsilon^{2}-\Delta^{2}-\Gamma^{2}+2 i \epsilon \Gamma}{2 e B}\right]
\end{aligned}
$$

so that Eq. 4.15) is rewritten as

$$
\begin{aligned}
D(\epsilon) & =\frac{N}{\pi^{2}}\left\{\Gamma\left[\ln \frac{\Lambda^{2}}{2 e B}-\operatorname{Re} \psi\left(\frac{\left|\epsilon^{2}-\Delta^{2}-\Gamma^{2}\right|-2 i \epsilon \Gamma}{2 e B}\right)-\frac{e B\left|\epsilon^{2}-\Delta^{2}-\Gamma^{2}\right|}{\left(\epsilon^{2}-\Delta^{2}-\Gamma^{2}\right)^{2}+4 \epsilon^{2} \Gamma^{2}}\right]\right. \\
& +\epsilon \operatorname{sgn}\left(\epsilon^{2}-\Delta^{2}-\Gamma^{2}\right)\left[\operatorname{Im} \psi\left(\frac{\left|\epsilon^{2}-\Delta^{2}-\Gamma^{2}\right|-2 i \epsilon \Gamma}{2 e B}\right)+\frac{2 e B \epsilon \Gamma}{\left(\epsilon^{2}-\Delta^{2}-\Gamma^{2}\right)^{2}+4 \epsilon^{2} \Gamma^{2}}\right] \\
& \left.+\pi \theta\left(\epsilon^{2}-\Delta^{2}-\Gamma^{2}\right) \frac{\epsilon \sinh (2 \pi \epsilon \Gamma / e B)-\Gamma \sin \left[\pi\left(\epsilon^{2}-\Delta^{2}-\Gamma^{2}\right) / e B\right]}{\cosh (2 \pi \epsilon \Gamma / e B)-\cos \left[\pi\left(\epsilon^{2}-\Delta^{2}-\Gamma^{2}\right) / e B\right]}\right\} .
\end{aligned}
$$

For small $\Gamma$ the main contribution comes from the last term in Eq. (4.19), thus DOS in the presence of $\Gamma$ can be represented in the form similar to Eq. (4.7a):

$$
\begin{aligned}
D(\epsilon) & =\frac{N}{2 \pi} \operatorname{sgn}(\epsilon) \frac{d}{d \epsilon}\left\{\theta ( \epsilon ^ { 2 } - \Delta ^ { 2 } - \Gamma ^ { 2 } ) \left[\epsilon^{2}-\Delta^{2}-\Gamma^{2}\right.\right. \\
& \left.\left.+2 e B \sum_{k=1}^{\infty} \frac{1}{\pi k} \sin \frac{\pi k\left(\epsilon^{2}-\Delta^{2}-\Gamma^{2}\right)}{e B} \exp \left(-\frac{2 \pi k|\epsilon| \Gamma}{e B}\right)\right]\right\} .
\end{aligned}
$$

Using now the formula

$$
\sum_{k=1}^{\infty} \frac{1}{k} e^{-k t} \sin k x=\tan ^{-1} \frac{\sin x}{e^{t}-\cos x}, \quad t>0,
$$

one can check that the last term in Eq. (4.19) is recovered. It is evident that oscillating part of DOS is contained in the sum over $k$ in Eq. (4.20).

\section{B. DOS in limiting cases}

Since the final expressions for DOS, Eq.4.15) and especially Eq.4.19) are rather lengthy, it is useful to consider a few simple limiting cases and compare them with the known results. First of all, it is easy to obtain from Eq. (4.15) that in the limit of zero field $B=0$ the DOS becomes

$$
D(\epsilon)=\frac{N}{\pi^{2}}\left[\Gamma \ln \frac{\Lambda}{\sqrt{\Gamma^{2}+(\epsilon-\Delta)^{2}}}+\Gamma \ln \frac{\Lambda}{\sqrt{\Gamma^{2}+(\epsilon+\Delta)^{2}}}+|\epsilon|\left(\frac{\pi}{2}+\tan ^{-1} \frac{\epsilon^{2}-\Delta^{2}-\Gamma^{2}}{2|\epsilon| \Gamma}\right)\right],
$$

and for $\Delta=0$, after restoring the prefactor $1 /\left(v_{F} v_{\Delta}\right)$ with the velocities $v_{F}$ and $v_{\Delta}$, it reduces to the DOS derived in Ref. [49].

As follows from Eq. (4.19), the DOS at zero energy, but in the finite field is given by

$$
D(0)=\frac{N \Gamma}{\pi^{2}}\left[\ln \frac{\Lambda^{2}}{\Delta^{2}+\Gamma^{2}}-\Psi\left(\frac{\Delta^{2}+\Gamma^{2}}{2 e B}\right)+\ln \frac{\Delta^{2}+\Gamma^{2}}{2 e B}-\frac{e B}{\Delta^{2}+\Gamma^{2}}\right] .
$$

The first term of Eq. (4.23) is nothing else but the zero energy DOS 4.22) in the absence of the magnetic field. The behavior of the DOS (4.23) can be now studied in various asymptotical regimes. For example, for $\Delta=0$ and $\Gamma \rightarrow 0$ we find

$$
D(0)=\frac{N}{\pi^{2}}\left[\frac{e B}{\Gamma}+\Gamma \ln \frac{\Lambda^{2}}{2 e B}\right]
$$


i.e., DOS is enhanced in the presence of the magnetic field if $\Gamma^{2} \ll e B$. In the opposite limit, $e B \ll \Gamma^{2}$ we have

$$
D(0)=\frac{N \Gamma}{\pi^{2}}\left[\ln \frac{\Lambda^{2}}{\Gamma^{2}}+\frac{(e B)^{2}}{3 \Gamma^{4}}\right] .
$$

On the other hand, for $\Delta \neq 0$ and $\Gamma \rightarrow 0$ we obtain

$$
D(0)=\frac{N \Gamma}{\pi^{2}}\left[\ln \frac{\Lambda^{2}}{2 e B}-\Psi\left(\frac{\Delta^{2}}{2 e B}\right)-\frac{e B}{\Delta^{2}}\right],
$$

so that nonzero gap regularizes $\Gamma \rightarrow 0$ divergence, which is present in Eq. (4.24).

\section{REPRESENTATION FOR THERMODYNAMIC POTENTIAL}

\section{A. General expressions}

All thermodynamic quantities such as the magnetization $\mathbf{M}$ and the number of electrons $N$ can be found from the grand thermodynamic potential $\Omega$, which in the relativistic case (see Refs. 25, 44]) can be written using the DOS as follows

$$
\Omega(T, \mu)=-T \int_{-\infty}^{\infty} d \epsilon D(\epsilon) \ln \left(2 \cosh \frac{\epsilon-\mu}{2 T}\right)
$$

with the DOS $D(\epsilon)$ given by Eq. (4.3). We assume everywhere that the volume (area) of the system is unity, so that Eq. (5.1) corresponds to the thermodynamic potential per unit volume. It is convenient to separate the vacuum contribution at $T=0, \mu=0$ in the thermodynamic potential. For that we write

$$
\begin{aligned}
\Omega(T, \mu) & =-T \int_{-\infty}^{\infty} d \epsilon D(\epsilon)\left[\ln \left(e^{\frac{\epsilon-\mu}{2 T}}+e^{\frac{\mu-\epsilon}{2 T}}\right)(\theta(\epsilon)+\theta(-\epsilon))\right] \\
& =-\frac{1}{2} \int_{-\infty}^{\infty} d \epsilon D(\epsilon)(\epsilon-\mu) \operatorname{sgn}(\epsilon)-T \int_{-\infty}^{\infty} d \epsilon D(\epsilon)\left[\ln \left(1+e^{\frac{\mu-\epsilon}{T}}\right) \theta(\epsilon)+\ln \left(1+e^{\frac{\epsilon-\mu}{T}}\right) \theta(-\epsilon)\right],
\end{aligned}
$$

or, using the evenness of the function $D(\epsilon)$ :

$$
\Omega(T, \mu)=-\int_{0}^{\infty} d \epsilon \epsilon D(\epsilon)-T \int_{-\infty}^{\infty} d \epsilon D(\epsilon)\left[\ln \left(1+e^{\frac{\mu-\epsilon}{T}}\right) \theta(\epsilon)+\ln \left(1+e^{\frac{\epsilon-\mu}{T}}\right) \theta(-\epsilon)\right] .
$$

The first (divergent) term in the last expression is the vacuum energy while the second one (convergent) is due to contributions of real quasiparticle excitations.

At zero temperature, $T=0$, we thus have

$$
\Omega(0, \mu)=\Omega(0, \mu=0)+\int_{0}^{|\mu|} d \epsilon D(\epsilon)(\epsilon-|\mu|) \equiv \Omega_{0}(0)+\tilde{\Omega}_{0}(\mu) .
$$

It is easy to see that the density of states is related to the thermodynamic potential at zero temperature

$$
D(\mu)=-\frac{d^{2} \Omega(T=0, \mu)}{d \mu^{2}} .
$$

As was already mentioned, the constant Landau level width approximation appears to be very useful simplification, viz. instead of calculating the potential (5.1) with $\Gamma \neq 0$, one can start from the potential $\Omega_{T}$,

$$
\Omega_{T}(\mu)=-T \int_{-\infty}^{\infty} d \epsilon D_{0}(\epsilon) \ln \left(2 \cosh \frac{\epsilon-\mu}{2 T}\right)
$$


where $D_{0}(\epsilon)$ is the DOS with zero level broadening $(\Gamma=0)$. Then the potential $\Omega$ at finite $\Gamma$ is obtained by convoluting $\Omega_{T}(\mu)$ with the distribution function $P_{\Gamma}(\omega)$. The statement that the level broadening is equivalent to considering the distribution of the chemical potentials was proven in Ref. 48] and we adopt here its derivation for the relativistic thermodynamic potential Eq.(5.1). Indeed, if the levels are broadened, then the density of states is obtained as a convolution of $D_{0}(\epsilon)$ with the probability distribution $P_{\Gamma}(\epsilon)$ of energies $\epsilon$ :

$$
\begin{aligned}
\Omega(T, \mu) & =-T \int_{-\infty}^{\infty} d \omega d \epsilon P_{\Gamma}(\omega-\epsilon) D_{0}(\omega) \ln \left(2 \cosh \frac{\epsilon-\mu}{2 T}\right)=-T \int_{-\infty}^{\infty} d \omega d \epsilon P_{\Gamma}(\omega) D_{0}(\epsilon) \ln \left(2 \cosh \frac{\epsilon-\omega-\mu}{2 T}\right) \\
& =-T \int_{-\infty}^{\infty} d \omega d \epsilon P_{\Gamma}(\omega-\mu) D_{0}(\epsilon) \ln \left(2 \cosh \frac{\epsilon-\omega}{2 T}\right)=\int_{-\infty}^{\infty} d \omega P_{\Gamma}(\omega-\mu) \Omega_{T}(\omega),
\end{aligned}
$$

where the potential $\Omega_{T}(\mu)$ is given by Eq.(5.6). If several damping effects occur together, the corresponding convolutions have to be carried out successively (the order is not essential).

The effect of finite temperature can also be included in this scheme by choosing the distribution function $P_{T}(z)$, which describes the temperature line broadening, equal to the negative derivative of the Fermi function, i.e.

$$
P_{T}(z)=-\frac{\partial n_{F}(z)}{\partial z}=\frac{1}{4 T \cosh ^{2} \frac{z}{2 T}}
$$

We now show that the thermodynamic potential Eq. (5.6) can be obtained as the convolution of $\Omega_{0}(\mu)$ with the distribution function $P_{T}$ given by Eq. (5.8)

$$
\Omega_{T}(\mu)=\int_{-\infty}^{\infty} d \omega P_{T}(\omega-\mu) \Omega_{0}(\omega)
$$

Since the vacuum contribution does not change under averaging over thermal and $\Gamma$ distributions, it is enough to prove this fact for the finite part $\tilde{\Omega}_{T}(\mu)$. Performing integration by parts we obtain:

$$
\tilde{\Omega}_{T}(\mu)=-T \int_{-\infty}^{\infty} d \epsilon D(\epsilon)\left[\ln \left(1+e^{\frac{\mu-\epsilon}{T}}\right) \theta(\epsilon)+\ln \left(1+e^{\frac{\epsilon-\mu}{T}}\right) \theta(-\epsilon)\right]=-\int_{-\infty}^{\infty} d \epsilon f_{F}(\epsilon) \int_{0}^{\epsilon} d x \operatorname{sgn}(x) D(x)
$$

where $f_{F}(\epsilon)$ is the relativistic thermal distribution function

$$
f_{F}(\epsilon)=\frac{\theta(\epsilon)}{1+e^{\frac{\epsilon-\mu}{T}}}+\frac{\theta(-\epsilon)}{1+e^{\frac{\mu-\epsilon}{T}}}
$$

which is also used in semiconductor physics. Integrating by parts for the second time, we obtain

$$
\tilde{\Omega}_{T}(\mu)=\int_{-\infty}^{\infty} \frac{\partial n_{F}(\epsilon-\mu)}{\partial \epsilon} W(\epsilon) d \epsilon
$$

where

$$
W(\epsilon)=\int_{0}^{\epsilon} d x \operatorname{sgn}(x) \int_{0}^{x} d y \operatorname{sgn}(y) D(y) .
$$

Since

$$
-\frac{\partial n_{F}(\epsilon)}{\partial \epsilon}=\frac{1}{4 T \cosh ^{2} \frac{\epsilon}{2 T}} \rightarrow \delta(\epsilon), \quad T \rightarrow 0
$$

we find that

$$
W(\mu)=-\tilde{\Omega}_{0}(\mu)
$$


hence

$$
\tilde{\Omega}_{T}(\mu)=\int_{-\infty}^{\infty} d \epsilon\left(-\frac{\partial n_{F}(\epsilon-\mu)}{\partial \epsilon}\right) \tilde{\Omega}_{0}(\epsilon) .
$$

The common effect of level broadening due to both temperature and damping effects can be written as successive convolutions

$$
\Omega(T, \mu)=\int_{-\infty}^{\infty} d \omega^{\prime} d \omega P_{\Gamma}\left(\omega^{\prime}-\mu\right) P_{T}\left(\omega-\omega^{\prime}\right) \Omega_{0}(\omega) .
$$

Hence for calculating thermodynamic quantities we need to know only the thermodynamic potential $\Omega_{0}(\omega)$ at zero temperature and zero width of levels that is directly expressed via the DOS $D_{0}(\epsilon)$, Eq. (4.7). Thus the knowledge of zero $T$ (and zero $\Gamma$ if the Landau levels have the same width) DOS is completely sufficient to write down the finite $T$ thermodynamic potential.

\section{B. Link with nonrelativistic thermodynamic potential and equation for chemical potential}

We start from the expression for the nonrelativistic thermodynamic potential (see, e.g. Ref. [1])

$$
\Omega_{\mathrm{NR}}(T, \mu)=-T \int_{-\infty}^{\infty} d \epsilon \mathcal{D}(\epsilon) \ln \left(1+e^{\frac{\mu-\epsilon}{T}}\right) .
$$

expressed in terms of the DOS $\mathcal{D}(\epsilon)$ for original nonrelativistic tight-binding Hamiltonians considered in Sec. [I Due to the complexity of the tight-binding spectrum, this DOS cannot be found explicitly for $B \neq 0$ and the only property of the DOS we need is that it is an even function of $\epsilon$.

The derivative of the thermodynamic potential (5.18) with respect to the chemical potential $\mu$,

$$
-\frac{\partial \Omega_{\mathrm{NR}}(T, \mu)}{\partial \mu}=\int_{-\infty}^{\infty} d \epsilon \mathcal{D}(\epsilon) n_{F}(\epsilon-\mu)=n
$$

determines the density of carriers $n$ in nonrelativistic many-body theory as a function of $T, B$ and $\mu$. On the other hand one can consider Eq. [5.19) as an equation for $\mu$ as a function of $T, B$ and $n$ which is typical for studies in a canonical ensemble. In Eq. (5.19) $n_{F}(\omega)=1 /[\exp (\omega / T)+1]$ is the usual Fermi function. At $T=0, \mu=0$ Eq. (5.19) gives the density of particles for a half-filled band.

$$
n_{0}=\int_{-\infty}^{\infty} d \epsilon \mathcal{D}(\epsilon) \theta(-\epsilon)
$$

so that the deviation of the particle density from $n_{0}$ is due to a finite temperature and nonzero chemical potential.

It is convenient to redefine the thermodynamic potential in order to have an explicit proportionality of $\mu$ to the density of free carriers. Thus we introduce

$$
\Omega^{\prime}(T, \mu)=\Omega_{\mathrm{NR}}(T, \mu)+\mu n_{0}=-\int_{-\infty}^{\infty} d \epsilon \mathcal{D}(\epsilon)\left[T \ln \left(1+e^{\frac{\mu-\epsilon}{T}}\right)-\mu \theta(-\epsilon)\right] .
$$

Inserting $1=\theta(\epsilon)+\theta(-\epsilon)$ before the logarithm, the last expression is rewritten in the form

$$
\Omega^{\prime}(T, \mu)=-\int_{0}^{\infty} d \epsilon \epsilon \mathcal{D}(\epsilon)-T \int_{-\infty}^{\infty} d \epsilon \mathcal{D}(\epsilon)\left[\ln \left(1+e^{\frac{\mu-\epsilon}{T}}\right) \theta(\epsilon)+\ln \left(1+e^{\frac{\epsilon-\mu}{T}}\right) \theta(-\epsilon)\right],
$$


where the first term gives the energy due to the half-filled zone. As is seen, $\Omega^{\prime}(T, \mu)$ acquires the form of a relativistic thermodynamic potential (compare with Eq. (5.3)). Its derivative with respect to $\mu$

$$
\begin{aligned}
\rho & =-\frac{\partial \Omega^{\prime}(T, \mu)}{\partial \mu}=\int_{-\infty}^{\infty} d \epsilon \mathcal{D}(\epsilon)\left[n_{F}(\epsilon-\mu) \theta(\epsilon)-\left[1-n_{F}(\epsilon-\mu)\right] \theta(-\epsilon)\right] \\
& =-\frac{1}{2} \int_{-\infty}^{\infty} d \epsilon \mathcal{D}(\epsilon) \tanh \frac{\epsilon-\mu}{2 T},
\end{aligned}
$$

determines the charge density of carriers or carrier imbalance, $\rho\left(\rho \equiv n-n_{0}=n_{+}-n_{-}\right.$, where $n_{+}$and $n_{-}$are the densities of "electrons" and "holes, respectively) as a function of $T$ and $B$ at fixed $\mu$. Note that now $\rho=0$ at $\mu=0$ and there is a symmetry with respect to the transformation $\mu \rightarrow-\mu$ and $\rho \rightarrow-\rho$. In the present paper, however, we will mostly use the canonical ensemble interpretation of Eq. (5.23), viz. as the equation for $\mu$ as a function of $T$ and $B$ at fixed $\rho$.

\section{ZERO TEMPERATURE THERMODYNAMIC POTENTIAL}

The zero temperature thermodynamic potential is given by Eq.(5.4), and for calculation of its vacuum contribution $\Omega_{0}(0)$ we refer to Appendix $\AA$ The thermodynamic potential with vacuum energy $\Omega_{0}(0)$ subtracted is given by the second term of Eq. (5.4)

$$
\tilde{\Omega}_{0}(\mu)=\int_{0}^{|\mu|} d \epsilon D_{0}(\epsilon)(\epsilon-|\mu|),
$$

where the DOS for $\Gamma=0$ case is given by Eq. (4.7). In what follows we choose for definiteness $\mu>0$. Calculating $\tilde{\Omega}_{0}(\mu)$ (see Appendix $\mathbb{B}$ for the detail) we obtain that it can be represented as a sum of regular and oscillating terms,

$$
\tilde{\Omega}_{0}(\mu)=\Omega_{r e g}(\mu)+\Omega_{o s c}(\mu),
$$

with $\Omega_{r e g}(\mu)$ expressed in terms of generalized zeta function

$$
\Omega_{r e g}(\mu)=-\frac{N}{2 \pi} \theta(\mu-\Delta)\left[\frac{1}{3} \mu\left(\mu^{2}-3 \Delta^{2}\right)-\Delta e B-(2 e B)^{3 / 2} \zeta\left(-\frac{1}{2}, 1+\frac{\Delta^{2}}{2 e B}\right)\right],
$$

and

$$
\Omega_{o s c}(\mu)=\frac{N(e B)^{3 / 2}}{2 \pi} \theta(\mu-\Delta) \sum_{k=1}^{\infty} \frac{1}{(\pi k)^{3 / 2}}\left[J_{1}(\pi k v) \cos (\pi k w)+J_{2}(\pi k v) \sin (\pi k w)\right],
$$

where the monotonic functions $J_{1,2}$ are defined by Eq. (B5). Writing the last expression for $\Omega_{\text {osc }}(\mu)$ we introduced new variables

$$
w=\frac{\mu^{2}-\Delta^{2}}{e B}, \quad v=\frac{\mu^{2}}{e B} .
$$

For small fields, $e B<<\mu^{2}$, we can use the asymptotic expansion (B7) for $J_{1}, J_{2}$ and represent $\Omega_{o s c}$ in terms of periodically continued Bernoulli polynomials defined in Eq. 4.9):

$$
\Omega_{o s c}(\mu)=\frac{N(e B)^{2}}{\pi^{3 / 2} \mu} \theta(\mu-\Delta) \sum_{n=0}^{\infty} \frac{\Gamma(n+1 / 2) B_{n+2}(w / 2)}{(n+2) !}\left(\frac{2 e B}{\mu^{2}}\right)^{n} .
$$

In particular, keeping the first few terms in the expansion we have

$$
\Omega_{o s c}(\mu) \simeq \frac{N(e B)^{2}}{2 \pi \mu} \theta(\mu-\Delta)\left[B_{2}\left(\frac{w}{2}\right)+\frac{e B}{3 \mu^{2}} B_{3}\left(\frac{w}{2}\right)+\frac{(e B)^{2}}{4 \mu^{4}} B_{4}\left(\frac{w}{2}\right)\right],
$$

where the explicit expressions for the Bernoulli polynomials $B_{1}-B_{4}$ are

$$
B_{1}(x)=x-1 / 2, \quad B_{2}(x)=x^{2}-x+1 / 6, \quad B_{3}(x)=x^{3}-3 x^{2} / 2+x / 2, \quad B_{4}(x)=x^{4}-2 x^{3}+x^{2}-1 / 30 .
$$

The terms oscillating with a magnetic field (Eqs. (6.6), (6.7)) represent small corrections to the nonoscillating part of the thermodynamic potential (Eq. (6.3)) at $e B \ll \mu^{2}$. Nevertheless, since $\Omega_{o s c}$ contains fast oscillating functions of the variable $\left(\mu^{2}-\Delta^{2}\right) / 2 e B$, after differentiating it over the magnetic field, the magnetization, $M=-\partial \Omega / \partial B$, acquires a large oscillating part (see Sec. VII] and Figs. 1 2 2). 


\section{A. The period of oscillations and its dependence on $\Delta$ for fixed $\mu$}

As it follows from the expressions (6.4), (6.6), the frequency of oscillations of the thermodynamic potential is equal to $w / 2=\left(\mu^{2}-\Delta^{2}\right) / 2 e B$. Therefore their period $\delta B$ satisfies the relationship

$$
\frac{\delta B}{B}=\frac{2 e B}{\mu^{2}-\Delta^{2}} \rightarrow \frac{\omega_{L}^{2}}{\mu^{2}-\Delta^{2}}, \quad \delta B \ll B,
$$

where $\omega_{L}$ is defined in Eq. (3.5). Using the estimate of $\omega_{L}$ for the DDW model made below Eq. (3.5), we obtain that for $\Delta=0, \mu=100 K$ and $B=1$ Tesla, the period of oscillation $\delta B=0.032$ Tesla, which is clearly within the detectable range of dHvA oscillation experiments and close to the estimate $\delta B \approx 0.05$ Tesla made in Ref. 35.

Repeating this estimate with $B=1$ Tesla for graphite with $\mu=200 K$ and $\Delta=0$, we get $\delta B=4$ Tesla which is very close to the observed value for period of the $\mathrm{SdH}$ oscillations in this material [18]. However, so large value of $\delta B$ clearly violates the condition $\delta B \ll B$ implied to obtain Eq. [6.9), and for the case of graphite the period of MO is given by a more accurate expression

$$
\left|\frac{1}{e B_{i}}-\frac{1}{e B_{i+1}}\right|=\frac{2}{\mu^{2}-\Delta^{2}}=\frac{2 \pi}{A},
$$

where $B_{i}$ and $B_{i+1}$ are the values of the magnetic field in two adjacent minima. In particular, for graphite again using for an estimate $\mu=200 \mathrm{~K}$ and $\Delta=0$ we obtain $\left|1 / B_{i}-1 / B_{i+1}\right|=8 \mathrm{Tesla}^{-1}$. Writing the last equality in Eq. [6.10), we introduced the area of an extremal cross section of the Fermi surface, $A=\pi\left(\mu^{2}-\Delta^{2}\right)$ as it is usually done. Thus, contrary to conventional systems with quadratic dispersion law and the period of $\mathrm{MO} \sim 1 / \mu$, the period of $\mathrm{MO}$ in the systems with linear dispersion (for $\Delta=0$ ) is $\sim 1 / \mu^{2}$ as seen in Fig. [1]

Making above estimates we have assumed everywhere that $\Delta=0$. However, as it is clear from Eqs. (6.9) and (6.10), the opening of the gap $\Delta$ increases the period of MO. In particular, for graphite even a rather small value of $\Delta$, e.g. $\Delta \leq 10^{-2} \mu$, would produce a sizeable $\sim 0.04$ Tesla change in the period of MO. The effect of the gap opening on the oscillations of the magnetization is shown in Fig. 2 This figure was obtained using the values of the parameters typical for graphite (see the caption). As one can see, the gap opening produces an observable change in the period of MO. Thus this method can be useful to test the realization of magnetic catalysis phenomenon [4] in graphite (see Ref. [42]), when the external magnetic field can induce the opening of an insulating gap $\Delta(B)$ in the relativistic spectrum of quasiparticle excitations. The crucial condition for the observation of the $\delta B$ increase caused by the gap opening is that the chemical potential $\mu$ itself does not change [50] as the gap $\Delta$ opens. Formally this condition corresponds to considering fixed $\mu$ in the grand canonical ensemble. However, if the experimental setup corresponds to the fixed carrier imbalance $\rho$, one can see from Eqs. (8.3) and (8.4) below that the entire difference $\mu^{2}-\Delta^{2}=2 \pi|\rho| / N$ adjusts to the strength of the applied field, so that the period of MO remains unchanged, making the gap detection impossible. Nevertheless, as we discuss at the end of Sec. VIIIC there is still a possibility of the gap detection if simultaneously with a frequency the amplitude of dHvA oscillations is measured.

As pointed out in Ref. [35], the estimate of $\delta B$ is not sufficient for the detectability of dHvA effect. In addition we should calculate the magnetization and estimate the magnitude of its oscillations.

\section{MAGNETIZATION}

The magnetization $M$ in the direction perpendicular to the plane should be calculated in the canonical ensemble, thus we need the Helmholtz free energy which is related to the thermodynamic potential as

$$
F(\rho, B)=\Omega(\mu(\rho, B), B)+\mu(\rho, B) \rho .
$$

Then

$$
M_{\rho}(\rho, B)=-\frac{\partial F(\rho, B)}{\partial B}=-\left.\frac{\partial \Omega(\mu, B)}{\partial B}\right|_{\mu=\mu(\rho, B)}=M_{\mu}(\mu(\rho, B), B),
$$

where $M_{\mu}$ is the magnetization obtained in the grand canonical ensemble at constant $\mu$ :

$$
M_{\mu}(\mu, B)=-\left.\frac{\partial \Omega(\mu, B)}{\partial B}\right|_{\mu=\mathrm{const}},
$$

and we used that $\partial \Omega(\mu, B) / \partial \mu=-\rho$. Formally, the magnetization calculated in the canonical ensemble has the same form as the magnetization calculated in the grand canonical ensemble, but we have to take into account oscillations 
of $\mu$ in the system with fixed $\rho$. In the present paper we restrict ourselves in what follows by studying MO in the limit $\omega_{c} \ll \epsilon_{F}$, so that for fixed $\rho$, as shown in Sec. VIIIA one can neglect the oscillations of $\mu(B)$.

The thermodynamic potential consists of the sum of three terms, the vacuum energy (A4), regular (6.3) and oscillating (6.4) parts. We begin with the calculation of the part of the magnetization which is due to the vacuum energy Eq. (A44).

As one could see from Eq. (5.22), this energy corresponds to the energy of the half-filled zone. Thus this case is also interesting from physical point of view [12. Considering firstly the limit $\Delta=0$, we obtain from Eq. (A5) that the magnetization

$$
M(\Delta=0, \mu=0)=-\frac{3 N \zeta(3 / 2)}{4 \sqrt{2} \pi^{2}} e^{3 / 2} \sqrt{B}
$$

(note the diamagnetic character of the vacuum contribution in magnetization). The $\sim \sqrt{B}$ dependence of the magnetization at $\mu=0$ implies that the susceptibility $\chi=\partial M / \partial B \propto B^{-1 / 2}$ diverges at zero field raising a question about the stability of a homogeneous state described by effective Lagrangian (2.16). As discussed in Refs. 12, 35] in the case of the OAF (DDW) this instability disappears if a coupling between layers is included, or if finite temperature and/or chemical potential is included. The instability is also removed in the presence of the nonzero gap $\Delta$. Indeed, using the asymptotics for zeta function

$$
\zeta(s, 1+a) \approx \frac{a^{1-s}}{s-1}-\frac{1}{2 a^{s}}+\frac{s}{12 a^{1+s}}
$$

for $a \gg 1$, we obtain from Eq. (A4) that

$$
M(\Delta, \mu=0)=-\frac{N e}{6 \pi} \frac{e B}{\Delta}, \quad e B \ll \Delta^{2} .
$$

Thus the opening of the gap removes the singularity at $B=0$ in the susceptibility.

Now we turn to the case $\mu>\Delta$ when in addition to the vacuum contribution two more terms appear in the thermodynamic potential, the regular $\Omega_{r e g}(\mu)$ and oscillating $\Omega_{o s c}(\mu)$ parts, that at $T=0$ are given by Eq. (6.3) and Eq. (6.4). As one can easily see, for $\mu>\Delta$ field dependent terms in Eq. 6.3) coincide with the corresponding terms in the vacuum energy (A4) up to a sign, so that their total contribution to the magnetization is zero. Therefore, only the oscillating term of the thermodynamic potential given by Eq. (6.4) contributes to the magnetization.

In the small field limit, $e B \ll \mu^{2}$ differentiating the potential (6.6) we obtain

$$
M(\Delta, \mu)=-\frac{N e \mu \theta(\mu-\Delta)}{2 \pi^{3 / 2}} \sum_{n=0}^{\infty} \frac{\Gamma(n+1 / 2)}{(n+1) !}\left[B_{n+2}\left(\frac{w}{2}\right)-B_{n+1}\left(\frac{w}{2}\right) \frac{w}{2}\right]\left(\frac{2 e B}{\mu^{2}}\right)^{n+1}
$$

where we used the relation $\partial B_{n}(z) / \partial z=n B_{n-1}(z)$. Eq. (17.7) is the generalization to the relativistic spectrum of the formula for the magnetization obtained by Shoenberg [1]. For example, keeping first few terms in the expansion (7.7) we have

$$
M(\Delta=0, \mu)=\frac{N e \mu}{2 \pi}\left[B_{1}\left(\frac{v}{2}\right)-\frac{3}{2} \frac{e B}{\mu^{2}} B_{2}\left(\frac{v}{2}\right)-\frac{1}{2} \frac{e^{2} B^{2}}{\mu^{4}} B_{3}\left(\frac{v}{2}\right)+O\left(\frac{e^{3} B^{3}}{\mu^{6}}\right)\right],
$$

where the explicit expressions for the Bernoulli polynomials are given in Eq. (6.8). We recall that the Bernoulli polynomials used here depend on the fractional part of their argument so that magnetization is oscillating function with the period $\delta B$ given by Eq. (6.9) and weakly varying amplitude. The dependence of the magnetization on the inverse field $B^{-1}$ is presented in Figs. [1 and 2 computed on the basis of Eq. (7.7). The dependence $M\left(B^{-1}\right)$ has a clear saw-tooth like shape showing that for the considered range of the parameters, it is indeed convenient to represent the oscillations in terms of the Bernoulli polynomials.

\section{INFLUENCE OF IMPURITIES AND TEMPERATURE ON MAGNETIC OSCILLATIONS}

\section{A. Thermodynamic potential $\tilde{\Omega}(\mu)$ and equation for $\mu$ in the presence of scattering}

To obtain the equation for the chemical potential it is convenient to use the DOS expressed as in Eq. (4.16), which allows one to write down the thermodynamic potential (6.1), including the effect of impurity scattering, as

$$
\tilde{\Omega}_{0}(\mu)=-\frac{N \mu^{2}}{2 \pi^{2}} \Gamma \ln \frac{\Lambda^{2}}{2 e B}-\frac{N e B}{\pi^{2}} \int_{0}^{\mu} d \epsilon \operatorname{Im}\left[\ln \Gamma\left(\frac{\Delta^{2}-(\epsilon+i \Gamma)^{2}}{2 e B}\right)+\frac{1}{2} \ln \left(\frac{\Delta^{2}-(\epsilon+i \Gamma)^{2}}{2 e B}\right)\right]
$$


Correspondingly, Eq. (5.23) for the carrier imbalance at $T=0$ and finite $\Gamma$ takes the form

$$
\rho=-\frac{\partial \tilde{\Omega}_{0}(\mu)}{\partial \mu}=\frac{N \mu}{\pi^{2}} \Gamma \ln \frac{\Lambda^{2}}{2 e B}+\frac{N e B}{\pi^{2}} \operatorname{Im}\left[\ln \Gamma\left(\frac{\Delta^{2}-(\mu+i \Gamma)^{2}}{2 e B}\right)+\frac{1}{2} \ln \left(\frac{\Delta^{2}-(\mu+i \Gamma)^{2}}{2 e B}\right)\right] .
$$

For small $\Gamma \ll \mu, \Delta$, one can derive from Eq. (8.2) a more simple expression

$$
\epsilon_{F}^{2}=\mu^{2}+\frac{2 e B}{\pi} \tan ^{-1} \frac{\sin \left[2 \pi\left(\mu^{2}-\Delta^{2}\right) /(2 e B)\right]}{\exp [2 \pi \mu \Gamma /(e B)]-\cos \left[2 \pi\left(\mu^{2}-\Delta^{2}\right) /(2 e B)\right]}, \quad \mu^{2}>\Delta^{2},
$$

where we have introduced the Fermi energy $\epsilon_{F}$, counted from the edge of the gap $\Delta$, by means of the relation $(N / 2 \pi)\left(\epsilon_{F}^{2}-\Delta^{2}\right)=\rho$. Note that Eq. [8.3] can also be derived directly from the relationship $\rho=\int_{0}^{\mu} d \epsilon D_{0}(\epsilon)$ and Eq. (4.20) using the sum (4.21).

In general, as mentioned in the Introduction, both fixed $\mu$ and fixed $\rho$ cases are possible. For example, fixing $\mu$ in Eq. (8.2) one can study the oscillations of the carrier imbalance $\rho$ as a function of the field $B$. Eq. (8.3) can be considered as the relativistic analog of the corresponding relation between $\mu$ and $\epsilon_{F}$ in nonrelativistic case 7]. One can deduce formal "nonrelativistic" limit from Eq. (8.3) if one defines the nonrelativistic Fermi energy $\epsilon_{F(n r)}=\pi \rho / N \Delta$ and corresponding "nonrelativistic" chemical potential $\mu=\mu_{n r}+\Delta$ and consider $\mu_{n r}<<\Delta$. This nonrelativistic limit is, in fact, irrelevant for MO considered in the present paper, because "relativistic" theory studied here emerges as an effective theory for nonrelativistic models described in Sec. III so that it is meaningless to consider its nonrelativistic limit.

On the other hand, for the magnetization the case of a fixed carrier imbalance $\rho$ is more natural, and therefore oscillations of the chemical potential have to be taken into account by solving Eq. (8.3) for $\mu(B)$. Generally, this nonlinear equation cannot be solved analytically without further approximations, but for $\epsilon_{F}^{2} \gg e B$ the second term in Eq. (8.3) represents a small correction to the constant value of the chemical potential so that we obtain by iterations an approximate solution

$$
\mu^{2}=\epsilon_{F}^{2}-\frac{2 e B}{\pi} \tan ^{-1} \frac{\sin \left[2 \pi\left(\epsilon_{F}^{2}-\Delta^{2}\right) /(2 e B)\right]}{\exp \left[2 \pi \epsilon_{F} \Gamma /(e B)\right]-\cos \left[2 \pi\left(\epsilon_{F}^{2}-\Delta^{2}\right) /(2 e B)\right]} .
$$

It is plotted in Fig. [3 for the model parameters typical for graphite. As expected, these oscillations have saw-tooth like shape and the sharpness of the teeth depends on the value of $\Gamma$. One can also anticipate that the relative amplitude of the oscillations decreases as the Fermi energy $\epsilon_{F}$ increases and the field $B$ decreases. This is indeed seen in Fig. 3 Yet in 2D the chemical potential oscillations are rather strong even for large $\epsilon_{F}$, due to the particular form of Eq. (8.3) that turns out to be different from its 3D counterpart.

In spite of the smallness of the second term in Eq. 8.4. it is important to take into account the difference of $\mu$ and $\epsilon_{F}$ in the arguments of oscillating functions (in the magnetization, for example) in 2D, because it alters the phase of oscillations. The difference can be neglected if the additional condition $2 \pi \mu \Gamma \gtrsim e B$ is valid (see discussion about the importance of the chemical potential oscillations in Ref. [8]). This problem, however, is beyond the scope of the present work.

\section{B. Thermodynamic potential $\Omega_{o s c}(\mu)$ and magnetization $M_{o s c}$ in the presence of scattering}

We return now to the oscillating part of the thermodynamic potential (6.1) including the effect of impurity scattering. The oscillating part can be written (see Appendix [C] for details) as

$$
\Omega_{o s c}(\mu, \Gamma)=\frac{N(e B)^{3 / 2}}{2 \pi} \theta\left(\mu^{2}-\Delta^{2}-\Gamma^{2}\right) \sum_{k=1}^{\infty} \frac{1}{(\pi k)^{3 / 2}}\left[J_{1}(\pi k v, \pi k \gamma) \cos (\pi k w)+J_{2}(\pi k v, \pi k \gamma) \sin (\pi k w)\right]
$$

where the functions of two arguments $J_{1}(\mathrm{p}, \mathrm{r})$ and $J_{2}(p, r)$ that generalize the functions $J_{1}$ and $J_{2}$ defined in Eq. (BB5), are defined in Eq. (C4) and the variables $w, u$ and $\gamma$ are

$$
w=\frac{\mu^{2}-\Delta^{2}-\Gamma^{2}}{e B}, \quad u=\frac{\Delta^{2}+\Gamma^{2}}{e B}, \quad \gamma=\frac{\Gamma^{2}}{e B} .
$$

Note that the variable $w$ (and also $u$ ) that was defined in Sec. VI by Eq. (6.5) is redefined in the presence of scattering in accordance with Eq. (8.6). As before the frequency of $\mathrm{MO}$ is given by $w / 2$, so that it would diminish in the presence of impurities if we considered fixed $\mu$ case. This decrease of the MO frequency for fixed $\mu$ is specific for the present 
relativistic model, while in the nonrelativistic case the MO frequency remains intact even in the presence of impurities. The origin of the difference between relativistic and nonrelativistic cases can be traced back to the difference in zero field DOS for these cases.

For $\Gamma, \sqrt{e B}<<\mu$ keeping the leading term in asymptotic expansions (C8), (C) (this is the first term in Eq. (C8) only), we obtain from Eq. (8.5) (omitting theta function)

$$
\Omega_{o s c}(\mu, \Gamma)=\frac{N(e B)^{2}}{2 \pi \mu} \sum_{k=1}^{\infty} \frac{\cos (\pi k w)}{(\pi k)^{2}} \exp \left(-2 \pi k \frac{\mu \Gamma}{e B}\right), \quad \mu>0 .
$$

It is easy to check that for $\Gamma=0$ Eq. (8.7) reduces to the expression that follows directly from Eq. (6.4) if one substitutes the first term of the expansion (B7), $J_{1}(p) \approx 1 / \sqrt{p}$. This comparison with Eq. (6.4) allows to identify in Eq. (8.7) the Dingle factor

$$
R_{D}(k, \mu)=\exp \left(-2 \pi k \frac{\mu \Gamma}{e B}\right)
$$

which describes reduction of the amplitude of $k$-th harmonic due to the electron scattering. As is seen, the finite amount of impurities leads to smoothing of the oscillations. The factor [8.8) is a generalization of the Dingle harmonic damping

$$
R_{D}^{\mathrm{NR}}(k) \propto \exp \left(-2 \pi k \frac{\Gamma}{\omega_{c}}\right)
$$

for the nonrelativistic case, usually written in terms of the nonrelativistic cyclotron frequency $\omega_{c}$. Comparing Eqs. (8.8) and (8.9), one can notice two facts. First one was already discussed in Sec. IIII viz. in the relativistic case $R_{D}$ can be larger than it would be for the nonrelativistic case for the same field for $\mu \lesssim m v_{F} v_{2}=\omega_{L}^{2} /\left(2 \omega_{c}\right)$, so that the condition for the observation of $\mathrm{MO}$ in the relativistic case is less strict. In particular, for graphite this condition remains valid for $\mu \lesssim m_{e} v_{F}^{2} \approx 6 \times 10^{4} \mathrm{~K}$. Secondly, further increase of $\mu$ makes MO unobservable, contrary to the nonrelativistic case, when $R_{D}$ does not depends on the chemical potential $\mu$.

Calculating the magnetization (7.3) and keeping only dominant terms, we obtain from Eq. (8.7)

$$
\begin{aligned}
M_{o s c} & =-\frac{N\left(\mu^{2}-\Delta^{2}-\Gamma^{2}\right)}{2 \pi \mu} \sum_{k=1}^{\infty} \frac{\sin (\pi k w)}{\pi k} e^{-2 \pi k \frac{\mu \Gamma}{e B}} \\
& =-\frac{N\left(\mu^{2}-\Delta^{2}-\Gamma^{2}\right)}{2 \pi \mu} \tan ^{-1} \frac{\sin \left[2 \pi\left(\mu^{2}-\Delta^{2}-\Gamma^{2}\right) /(2 e B)\right]}{\exp [2 \pi \mu \Gamma /(e B)]-\cos \left[2 \pi\left(\mu^{2}-\Delta^{2}-\Gamma^{2}\right) /(2 e B)\right]},
\end{aligned}
$$

where in the second equality we used Eq. (4.21). We will show now that the oscillating part of the magnetization $M_{o s c}$ is directly related to the oscillating part of the chemical potential.

Comparing the result Eq. (8.10) for $M_{\text {osc }}$ with the solution for chemical potential (8.4), one can notice that the oscillating part of the magnetization is directly proportional to the oscillating part of $\mu^{2}$ :

$$
M_{o s c}=\frac{N\left(\epsilon_{F}^{2}-\Delta^{2}\right)}{4 e B \epsilon_{F}}\left(\mu^{2}\right)_{o s c}=\frac{\pi \rho}{2 e B} \frac{\left(\mu^{2}\right)_{o s c}}{\epsilon_{F}}
$$

(this is obvious if one neglects small terms $\Gamma^{2}$ in the trigonometric functions in Eq. (8.10)). This proportionality can be in fact seen when Fig. 3 (plotted for finite $\Gamma$ ) is compared with Figs. 1 and 2 (plotted for $\Gamma=0$ ). Note that unlike nonrelativistic case [7] the magnetization is proportional to $\mu_{o s c}^{2}$, not $\mu_{o s c}$. If the ratios $\epsilon_{F}^{2} / e B$ and $2 \pi \mu \Gamma / e B$ are small, the oscillating part of the chemical potential under trigonometric functions in Eq. (8.10) leads to additional frequencies in the Fourier spectrum of the magnetization. In its turn this changes the harmonics amplitudes compared with the LK like formula Eq.(8.10). In the non-relativistic case this was demonstrated in Ref. 8, 50, 51].

\section{Calculation of $\Omega_{o s c}(\mu)$ for $T \neq 0$}

Now we consider the effect of finite temperature on the oscillations of the thermodynamic potential that can be calculated from the zero temperature potential (8.5) using the convolution property Eq. (5.9). For that it is convenient to rewrite the expression (8.5) in the form

$$
\Omega_{o s c}(\mu, \Gamma)=-\frac{N(e B)^{3 / 2}}{2 \pi^{3 / 2}} \theta\left(\mu^{2}-\Delta^{2}-\Gamma^{2}\right) \sum_{k=1}^{\infty} \frac{1}{(\pi k)^{3 / 2}} \operatorname{Im}\left[e^{-\frac{i \pi}{4}} \int_{0}^{\infty} \frac{d t e^{-i(\pi k v) t+\frac{i \pi k \gamma}{t}}}{\sqrt{t}(t+1)} e^{-i \pi k w}\right]
$$


where we used that the functions $J_{1}(p, r)$ and $J_{2}(p, r)$ can be represented as - Im and Re parts of the same function

$$
\sqrt{\pi} J_{1}(p, r)=-\operatorname{Im} \int_{0}^{\infty} \frac{d t e^{-p t-r / t}}{\sqrt{t}(t+i)}, \quad \sqrt{\pi} J_{2}(p, r)=\operatorname{Re} \int_{0}^{\infty} \frac{d t e^{-p t-r / t}}{\sqrt{t}(t+i)},
$$

and rotated the integration contour to the imaginary axis. Hence

$$
\begin{aligned}
\Omega_{o s c}(T, \mu, \Gamma) & =-\frac{N(e B)^{3 / 2}}{2 \pi^{3 / 2}} \sum_{k=1}^{\infty} \frac{1}{(\pi k)^{3 / 2}} \operatorname{Im}\left[e^{-\frac{i \pi}{4}} \int_{0}^{\infty} \frac{d t e^{i \pi k \gamma / t}}{\sqrt{t}(t+1)} \int_{-\infty}^{\infty} \frac{d \epsilon}{4 T \cosh ^{2} \frac{\epsilon-\mu}{2 T}}\right. \\
& \left.\times \theta\left(\epsilon^{2}-\Delta^{2}-\Gamma^{2}\right) \exp \left(-\frac{i t \pi k \epsilon^{2}}{e B}-i \pi k \frac{\epsilon^{2}-\Delta^{2}-\Gamma^{2}}{e B}\right)\right] .
\end{aligned}
$$

At low temperatures, $T \rightarrow 0$, after making a shift $\epsilon \rightarrow \epsilon+\mu$ and changing the variable of integration $\epsilon \rightarrow 2 T \epsilon$, the integral over $\epsilon$ gives (neglecting $T^{2}$ term in the exponent)

$$
\int_{-\infty}^{\infty} \frac{d \epsilon}{\cosh ^{2} \epsilon} e^{-\frac{i \pi k}{e B}(t+1) 4 T \mu \epsilon}=2 \int_{0}^{\infty} \frac{d \epsilon}{\cosh ^{2} \epsilon} \cos \left(\frac{4 T \mu(t+1) \pi k}{e B} \epsilon\right)=\frac{4 \pi^{2} k T \mu(t+1)}{e B \sinh \frac{2 \pi^{2} k T \mu(t+1)}{e B}},
$$

where we used the formula (3.982.1) from Ref. [52]. We obtain

$$
\begin{aligned}
\Omega_{o s c}(T, \mu, \Gamma) & =-\frac{N(e B)^{1 / 2} T \mu}{\sqrt{\pi}} \theta\left(\mu^{2}-\Delta^{2}-\Gamma^{2}\right) \sum_{k=1}^{\infty} \frac{1}{(\pi k)^{1 / 2}} \\
& \times \operatorname{Im}\left[e^{-\frac{i \pi}{4}} e^{-i \pi k w} \int_{0}^{\infty} \frac{d t}{\sqrt{t}} e^{-\frac{i \pi k t \mu^{2}}{e B}+\frac{i \pi k \gamma}{t}} \frac{1}{\sinh \frac{2 \pi^{2} k T \mu(t+1)}{e B}}\right] .
\end{aligned}
$$

Nonoscillating factor in the integrand has a maximum at $t=0$, thus we can take $\sinh$ evaluated at $t=0$ while the remaining integral over $t$ is evaluated by means of Eqs. (C6), (C7). We finally get (omitting again theta function)

$$
\begin{aligned}
\Omega_{o s c}(T, \mu, \Gamma) & =N e B T \sum_{k=1}^{\infty} \frac{\cos (\pi k w)}{\pi k} \frac{1}{\sinh \frac{2 \pi^{2} k T \mu}{e B}} \exp \left[-2 \pi k \frac{\mu \Gamma}{e B}\right] \\
& =\frac{N(e B)^{2}}{2 \pi \mu} \sum_{k=1}^{\infty} \frac{\cos (\pi k w)}{(\pi k)^{2}} R_{T}(k, \mu) R_{D}(k, \mu),
\end{aligned}
$$

where we introduced the temperature amplitude factor

$$
R_{T}(k, \mu)=\frac{2 \pi^{2} k T \mu /(e B)}{\sinh \frac{2 \pi^{2} k T \mu}{e B}},
$$

which is a "relativistic" equivalent of the temperature reduction factor of the famous LK formula. Clearly, since $R_{T}(k, \mu) \rightarrow 1$ for $T \rightarrow 0$, Eq. (8.17) reduces to Eq. (8.7) and for finite $T$ the thermal broadening causes a reduction of the MO amplitude with respect to $T=0$ case. This becomes more evident if we consider the limit $B \rightarrow 0$, so that the function $1 / \sinh \left[2 \pi^{2} k T \mu /(e B)\right] \sim \exp \left[-2 \pi^{2} k T \mu /(e B)\right]$. Comparison of this exponent with Eq. (8.8) allows one to introduce the Dingle temperature

$$
T_{D}=\frac{\Gamma}{\pi}
$$

so that a reduction of amplitude due to quasiparticles scattering from impurities can be interpreted as leading to an effective rise of a temperature from true temperature $T$ to $T+T_{D}$, i.e., as if the system could not be cooled below a minimal temperature $T_{D}$. Note that while the Dingle factors (8.8) and (8.9) are different, the value of $T_{D}$ itself is the same for relativistic and nonrelativistic cases. Comparing Eq. 8.17) with the corresponding expression (15) for $\Omega_{o s c}$ in $2 \mathrm{D}$ from Ref. [] (this expression contains also a spin factor $R_{s}$ which is not considered in our work), one can notice that relativistic and nonrelativistic cases differ only by the factor $(-1)^{k}$ that appears due to the presence of the zero energy, $E_{0}=\omega_{c} / 2$ for the nonrelativistic Landau levels. 
It is seen from Eq. (8.17) that the conditions for observation of dHvA oscillations can be formulated as an inequality for the strength of magnetic field:

$$
\frac{\mu^{2}-\Delta^{2}}{2} \gtrsim e B \gtrsim \max \left\{2 \pi^{2} T \mu, 2 \pi^{2} T_{D} \mu\right\}
$$

The first inequality is necessary in order to have at least one oscillation. It implies physically that the field must not be so high that all fermions are in the lowest Landau level, i.e. the filling factor has to be bigger than one. The second inequality guarantees that the amplitude of oscillations is not exponentially suppressed, and for that the magnetic field must be strong enough to make spacing between Landau levels bigger or of the order of the thickness of the thermal layer times the Fermi energy.

The dependence of the magnetization (7.3) on $B^{-1}$ is obtained by differentiation of Eq. (8.17) and the results of numerical computations are shown in Figs. 4 and 5 They illustrate the above mentioned facts that the MO are smoothed as the temperature and/or the chemical potential rise. Obviously the increase of $\Gamma$ gives the same result. Moreover, one can clearly see the damping of dHvA amplitude as the field $B$ decreases. It is known that the damping of dHvA oscillation amplitude as function of several parameters is commonly used to extract system parameters such as Dingle temperature and the effective electron mass. However, these methods of analysis depend on the applicability of the LK theory. Assuming that the relativistic generalization of the LK theory considered here is valid at least for $\omega_{L} \ll \epsilon_{F}$, we suggest that, using Eqs. (8.10) and (8.17), one can extract both the value of $\mu$ from the damping and the difference, $\mu^{2}-\Delta^{2}$ from the frequency of dHvA oscillations. The knowledge of these two parameters allows to obtain the value of the gap $\Delta$ even for the fixed carrier imbalance $\rho$.

\section{CONCLUSIONS}

In this paper we have studied magnetic oscillations in thermodynamic quantities such as DOS, thermodynamic potential and magnetization in planar systems with the relativistic Dirac-like spectra for quasiparticle excitations. The attention was mainly paid to the regime, where the calculations in canonical (fixed $\rho$ ) and grand canonical (fixed $\mu$ ) ensembles give equivalent results. Our main results can be summarized as follows.

(1) We have obtained analytical expressions that describe MO in the DOS given by Eqs. 4.7), (4.19) for zero width $\Gamma=0$ (no impurities) and Eq. (4.20) for finite width $\Gamma$ for Landau's levels.

(2) For $\Gamma=0$, we have expressed the MO in thermodynamic potential, Eq. (6.6) and magnetization, Eq. (17.7) as a series in the periodically continued Bernoulli polynomials. This representation turns out to be useful for $2 \mathrm{D}$ case when the oscillations have saw-tooth like shape. In the limit $\omega_{L} \ll \mu$ it is sufficient to consider only first few terms of the series.

(3) For finite impurity scattering rate $\Gamma$ and temperature we have obtained the thermodynamic potential 8.17) and represented it in terms of the Dingle factor $R_{D}$, Eq. (8.8), and the temperature amplitude factor $R_{T}$, Eq. (8.18), as it is usually done in Lifshits-Kosevich theory. The spin factor, $R_{s}$, can be calculated similarly if the Zeeman term is included in the model.

(4) For finite $\Gamma$, we have derived also the equation (8.2) for the chemical potential. Its solution for fixed carrier imbalance exhibits oscillations of $\mu$ as a function of the magnetic field. It is shown that the oscillating part of the magnetization is in fact directly proportional to the oscillating part of the chemical potential (more precisely, to $\left.\left(\mu^{2}\right)_{\text {osc }}\right)$.

(5) On the basis of obtained formulas, we have discussed the possibility to detect a gap that may open in the spectrum of the Dirac-like quasiparticle excitations due to a nontrivial interaction between them.

One of the possible and necessary extensions of our results would be to study magnetic oscillations for the case when the consideration within the canonical ensemble is crucial, i.e. in the regime where the oscillations of the chemical potential described by Eq. (5.23) at fixed $\rho\left(\epsilon_{F}\right)$ cannot be neglected. The necessity of such an extension emerges from the fact that the oscillations of $\mu$ become important for $2 \pi \mu \Gamma \lesssim e B$ which is exactly the condition (8.20) for having large amplitude of the MO.

Another important extension would be to consider MO analytically in the transport quantities such as electrical and thermal conductivities. These oscillations were in fact seen in the numerical results presented in Ref. 29], but analytical results can be useful for comparison with the experiments [17, 18]. While for dHvA effect the condition $\rho=$ const is more natural, it is plausible that $\mathrm{SdH}$ effect can be measured under condition $\mu=$ const. This, as we have discussed in the paper, can be used for an experimental observation of the gap $\Delta$, especially if the gap depends on the applied field, $\Delta=\Delta(B)$.

All above-mentioned problems can be treated analytically due to the fact that the broadening of Landau levels has a Lorentzian shape and the impurity scattering rate $\Gamma$ (the width of this distribution) is assumed to be field and temperature independent. In fact, both these assumptions can be questioned, in particular for $2 \mathrm{D}$ systems with a 
linear dispersion. For example, the validity of the first assumption for $2 \mathrm{D}$ systems is now discussed in the literature (see Refs. in [8]). While the second assumption may well be valid in the low field regime, it is necessary to investigate its domain of validity and probably to consider also the dependence $\Gamma(B)$ 47] to access the high-field regime.

\section{ACKNOWLEDGMENTS}

We gratefully acknowledge N. Andrenacci and V.M. Loktev for stimulating discussions. One of us, S.G.Sh., thanks P. Esquinazi for a correspondence. This work was supported by the research project 2000-067853.02/1 of the Swiss NSF. V.P.G. acknowledges support from the Natural Sciences and Engineering Research Council of Canada. The work of V.P.G. was supported also by the SCOPES-projects 7UKPJ062150.00/1 and 7 IP 062607 of the Swiss NSF.

\section{APPENDIX A: CALCULATION OF VACUUM ENERGY $\Omega_{0}(\mu=0, B)$}

As mentioned above, the vacuum contribution does not change under averaging over thermal and $\Gamma$ distributions, so it is sufficient to calculate it at $T=0$ and in the absence of impurities. We calculate the vacuum term using the density of states in the form of Eq. (4.2)

$$
\Omega_{0}(\mu=0, B)=-\int_{0}^{\infty} d \epsilon \epsilon D_{0}(\epsilon)=-\frac{N e B}{2 \pi}\left[\Delta+2 \sum_{n=1}^{\infty} M_{n}\right]
$$

The sum over the Landau levels is divergent and to calculate it we use the representation

$$
\frac{1}{a^{s}}=\frac{1}{\Gamma(s)} \int_{0}^{\infty} d t t^{s-1} e^{-a t}
$$

to write

$$
\Omega_{0}(\mu=0, B)=-\frac{N e B}{2 \pi \Gamma(-1 / 2)} \int_{0}^{\infty} \frac{d t}{t^{3 / 2}} e^{-t \Delta^{2}}\left[1+2 \sum_{n=1}^{\infty} e^{-2 e B t}\right]=\frac{N}{4 \pi^{3 / 2}} \int_{1 / \Lambda^{2}}^{\infty} \frac{d t}{t^{5 / 2}} e^{-t \Delta^{2}} e B t \operatorname{coth}(e B t)
$$

where we introduced the ultraviolet cutoff $\Lambda$ as the bandwidth. The integral can be evaluated through the generalized zeta function

$$
\Omega_{0}(\mu=0, B)=-\frac{N}{2 \pi}\left[\frac{\Lambda \Delta^{2}}{\sqrt{\pi}}+\Delta e B+(2 e B)^{3 / 2} \zeta\left(-\frac{1}{2}, \frac{\Delta^{2}}{2 e B}+1\right)\right]+O\left(\frac{1}{\Lambda}\right)
$$

where the term $\sim \Lambda^{3}$ which does not depend on $\Delta$ and $B$ is omitted. The last expression coincides with the vacuum energy computed in the second paper in Ref. [44. Setting in Eq. A4 $\Delta=0$ and using the identity $\zeta(-1 / 2,1)=\zeta(-1 / 2)=-(1 / 4 \pi) \zeta(3 / 2)$ we obtain

$$
\Omega_{0}(\mu=0, B, \Delta=0)=\frac{N \zeta(3 / 2)}{2 \sqrt{2} \pi^{2}}(e B)^{3 / 2} .
$$

To compare Eq. (A5) with Eq. (15) of Ref. [12] and Eq. (10) of Ref. [35], one should restore the prefactor $1 /\left(v_{F} v_{D}\right)$ with the velocities $v_{F}, v_{D}$ and express them via the parameters $t$ and $D_{0}$ as done below Eq. (2.1). Note that the ground state energy increases with applied magnetic field, indicating diamagnetism.

\section{APPENDIX B: CALCULATION OF $\tilde{\Omega}_{0}(\mu)$}

Substituting Eq. (4.7a) in Eq. (6.1) and integrating by parts we obtain

$$
\tilde{\Omega}_{0}(\mu)=-\frac{N}{2 \pi} \int_{0}^{\mu} d \epsilon \theta\left(\epsilon^{2}-\Delta^{2}\right)\left[\epsilon^{2}-\Delta^{2}+2 e B \sum_{k=1}^{\infty} \frac{1}{\pi k} \sin \frac{\pi k\left(\epsilon^{2}-\Delta^{2}\right)}{e B}\right] \equiv \Omega_{0}^{(1)}(\mu)+\Omega_{0}^{(2)}(\mu),
$$


where

$$
\Omega_{0}^{(1)}(\mu)=-\frac{N}{6 \pi} \theta(\mu-\Delta)(\mu-\Delta)^{2}(\mu+2 \Delta)
$$

and

$$
\begin{aligned}
\Omega_{0}^{(2)}(\mu) & =-\frac{N e B}{\pi} \theta(\mu-\Delta) \sum_{k=1}^{\infty} \frac{1}{\pi k} \int_{\Delta}^{\mu} d \epsilon \sin \frac{\pi k\left(\epsilon^{2}-\Delta^{2}\right)}{e B} \\
& =-\frac{N(e B)^{3 / 2}}{2 \pi} \theta(\mu-\Delta) \sum_{k=1}^{\infty} \frac{1}{\pi k} \int_{0}^{w} \frac{d x \sin (\pi k x)}{\sqrt{x+u}} .
\end{aligned}
$$

Writing the last expression for $\Omega_{0}^{(2)}(\mu)$ we introduced new variables $w$ defined in Eq. 6.5) and $u=\Delta^{2} /(e B)$.

To extract explicitly the oscillations in the variable $w$ we rewrite the integral in Eq. (B3) as

$$
\begin{aligned}
& \int_{0}^{w} \frac{d x \sin (\pi k x)}{\sqrt{x+u}}=\int_{0}^{w} d x \sin (\pi k x) \frac{1}{\sqrt{\pi}} \int_{0}^{\infty} \frac{d t}{\sqrt{t}} e^{-t(x+u)} \\
& =\frac{1}{\pi \sqrt{k}}\left[\int_{0}^{\infty} \frac{d t e^{-t(\pi k u)}}{\sqrt{t}\left(t^{2}+1\right)}-\int_{0}^{\infty} \frac{d t e^{-t(\pi k v)}}{\sqrt{t}\left(t^{2}+1\right)} \cos (\pi k w)-\int_{0}^{\infty} \frac{d t \sqrt{t} e^{-t(\pi k v)}}{t^{2}+1} \sin (\pi k w)\right],
\end{aligned}
$$

where $v$ is defined in Eq. [6.5). The integrals in the last equation can be expressed in terms of the degenerate hypergeometric function $\Psi$ :

$$
\begin{aligned}
& \sqrt{\pi} J_{1}(p)=\int_{0}^{\infty} \frac{d t e^{-t p}}{\sqrt{t}\left(t^{2}+1\right)}=-\operatorname{Im} \int_{0}^{\infty} \frac{d t e^{-t p}}{\sqrt{t}(t+i)}=-\sqrt{\pi} \operatorname{Im}\left[i^{-1 / 2} \Psi\left(\frac{1}{2}, \frac{1}{2} ; i p\right)\right]=-\sqrt{\pi} \operatorname{Im}\left[\sqrt{p} \Psi\left(1, \frac{3}{2} ; i p\right)\right], \\
& \sqrt{\pi} J_{2}(p)=-\frac{\partial}{\partial p}\left[\sqrt{\pi} J_{1}(p)\right]=\int_{0}^{\infty} \frac{d t \sqrt{t} e^{-t p}}{t^{2}+1}=\operatorname{Re} \int_{0}^{\infty} \frac{d t e^{-t p}}{\sqrt{t}(t+i)}=\sqrt{\pi} \operatorname{Re}\left[\sqrt{p} \Psi\left(1, \frac{3}{2} ; i p\right)\right],
\end{aligned}
$$

where we used also the relationship [52]

$$
\Psi(a, b ; z)=z^{1-b} \Psi(a-b+1,2-b ; z) .
$$

One can readily see that the integrals $J_{1}, J_{2}$ are monotonic functions of their arguments and have the following asymptotic expansions

$$
J_{1}(p)=\frac{1}{\sqrt{\pi}} \sum_{n=0}^{\infty} \frac{(-1)^{n} \Gamma(2 n+1 / 2)}{p^{2 n+1 / 2}}, \quad J_{2}(p)=\frac{1}{\sqrt{\pi}} \sum_{n=0}^{\infty} \frac{(-1)^{n} \Gamma(2 n+3 / 2)}{p^{2 n+3 / 2}} .
$$

Thus for regular part of the potential we obtain

$$
\Omega_{r e g}(\mu)=-\frac{N}{6 \pi} \theta(\mu-\Delta)(\mu-\Delta)^{2}(\mu+2 \Delta)-\frac{N(e B)^{3 / 2}}{2 \pi} \theta(\mu-\Delta) \sum_{k=1}^{\infty} \frac{J_{1}(\pi k u)}{(\pi k)^{3 / 2}}
$$

while the oscillating part takes the form (6.4) written in Sec. V1] Eqs. (B8) and (6.4) were also derived in Ref. 24] using a different approach [53]. The sum in Eq. (B8) can be evaluated through the generalized zeta function in the following way

$$
\begin{aligned}
& \sum_{k=1}^{\infty} \frac{J_{1}(\pi k u)}{(\pi k)^{3 / 2}}=\frac{1}{\sqrt{\pi}} \sum_{k=1}^{\infty} \frac{1}{(\pi k)^{3 / 2}} \int_{0}^{\infty} \frac{d t e^{-\pi k u t}}{\sqrt{t}\left(t^{2}+1\right)}=\frac{1}{\sqrt{\pi}} \int_{0}^{\infty} \frac{d t e^{-u t}}{\sqrt{t}} \sum_{k=1}^{\infty} \frac{1}{t^{2}+(\pi k)^{2}} \\
& =\frac{1}{2 \sqrt{\pi}} \int_{0}^{\infty} d t t^{-3 / 2} e^{-u t}\left(\operatorname{coth} t-\frac{1}{t}\right)=-2^{3 / 2} \zeta\left(-\frac{1}{2}, 1+\frac{u}{2}\right)-u^{1 / 2}-\frac{2}{3} u^{3 / 2}
\end{aligned}
$$


which finally results in Eq. 6.3).

The oscillating part $\Omega_{o s c}(\mu)$ of the thermodynamic potential can be also represented in terms of the generalized zeta function. For that one should use $\Gamma(n+\alpha)=\int_{0}^{\infty} d s s^{n+\alpha-1} e^{-s}$ and perform summation over $n$ by means of the formula

$$
\sum_{n=0}^{\infty} \frac{x^{n}}{n !} B_{n}(y)=\frac{x e^{x y}}{e^{x}-1}, \quad|x|<2 \pi
$$

we obtain

$$
\sum_{n=2}^{\infty} \frac{\Gamma(n+\alpha) B_{n}(y)}{n !} x^{n}=\int_{0}^{\infty} d s s^{\alpha-1} e^{-s}\left[\frac{s x e^{s x y}}{e^{s x}-1}-1-s x B_{1}(y)\right] .
$$

Using now the formula 52

$$
\int_{0}^{\infty} \frac{x^{\nu-1} e^{-\mu x} d x}{1-e^{-\beta x}}=\frac{1}{\beta^{\nu}} \Gamma(\nu) \zeta\left(\nu, \frac{\mu}{\beta}\right), \quad \operatorname{Re} \mu>0, \operatorname{Re} \nu>0,
$$

we find

$$
\begin{aligned}
\Omega_{o s c}(\mu)= & -\frac{N \mu^{3}}{2 \pi} \theta(\mu-\Delta)\left\{\left(\frac{2 e B}{\mu^{2}}\right)^{3 / 2} \zeta\left(-\frac{1}{2}, 1+\frac{\mu^{2}}{2 e B}-\bmod \left[\frac{\mu^{2}-\Delta^{2}}{2 e B}\right]\right)\right. \\
& \left.+\frac{2}{3}-\frac{2 e B}{\mu^{2}}\left(\bmod \left[\frac{\mu^{2}-\Delta^{2}}{2 e B}\right]-\frac{1}{2}\right)\right\} .
\end{aligned}
$$

This expression involves a dependence on the nonanalytical fractional part mod $\left[\frac{\mu^{2}-\Delta^{2}}{2 e B}\right]$. In terms of the integer part $\left[\frac{\mu^{2}-\Delta^{2}}{2 e B}\right]$ the total thermodynamic potential (a regular plus oscillating parts) can be written as 25$]$

$$
\begin{aligned}
\tilde{\Omega}_{0}(\mu)=-\frac{N e B}{\pi} \theta(\mu-\Delta) & \left\{\mu\left[\frac{\mu^{2}-\Delta^{2}}{2 e B}\right]+\sqrt{2 e B} \zeta\left(-\frac{1}{2}, 1+\frac{\Delta^{2}}{2 e B}+\left[\frac{\mu^{2}-\Delta^{2}}{2 e B}\right]\right)\right. \\
& \left.+\frac{\mu-\Delta}{2}-\sqrt{2 e B} \zeta\left(-\frac{1}{2}, 1+\frac{\Delta^{2}}{2 e B}\right)\right\} .
\end{aligned}
$$

The last two terms are in fact cancelled by the zero chemical potential (vacuum) part of the thermodynamic potential Eq. (A4) (for $\mu>\Delta$ ).

In fact, the expression Eq. (B14) can be obtained much easier if one uses Eq. (4.2) and writes Eq. (6.4) as

$$
\tilde{\Omega}_{0}(\mu)=-\frac{N e B}{2 \pi}\left(\mu-\Delta+\sum_{n=0}^{\left[\frac{\mu^{2}-\Delta^{2}}{2 e B}\right]}\left(\mu-M_{n}\right)\right) .
$$

Using now the formula for the generalized zeta function

$$
\zeta(z, v+k)=\zeta(z, v)-\sum_{n=0}^{k-1}(n+v)^{-z}
$$

one immediately arrives at Eq. (B14). However, the oscillating property of $\tilde{\Omega}_{0}(\mu)$ is not so transparent in Eq. (B14) as it is in Eqs. (6.4) and (6.6). On the other hand, the expression for $\tilde{\Omega}_{0}(\mu)$ through the generalized zeta-function is convenient for studying large field behavior. Indeed, for $e B>>\Delta^{2}, \mu^{2}-\Delta^{2}$ we find from Eq.(B14) that in this limit

$$
\tilde{\Omega}_{0}(\mu) \simeq-\frac{N e B}{2 \pi} \theta(\mu-\Delta)(\mu-\Delta),
$$

which is precisely the contribution due to the lowest Landau level which is the only one to survive in the high field limit. Also, as is seen from Eq.(B14), the oscillating term (first zeta-function in curve brackets) is necessary to take into account even in the high field regime in order to cancel $(e B)^{3 / 2}$ term and obtain the correct linear term for $\tilde{\Omega}_{0}(\mu)$. 


\section{APPENDIX C: CALCULATION OF $\Omega_{o s c}(\mu)$ IN THE PRESENCE OF SCATTERING}

It turns out that due to the bad convergence of the integrals like (5.7) and (5.17) with the Lorentzian distribution $P_{\Gamma}(\omega)$ this calculation can be done more easily if we directly substitute the DOS (4.20) in Eq. 6.17) and generalize the calculation made in Sec. VI Choosing again for definiteness $\mu>0$, for the oscillating part of the thermodynamic potential we have

$$
\begin{aligned}
\Omega_{o s c}(\mu, \Gamma) & =-\frac{N e B}{\pi} \theta\left(\mu^{2}-\Delta^{2}-\Gamma^{2}\right) \sum_{k=1}^{\infty} \frac{1}{\pi k} \int_{\sqrt{\Delta^{2}+\Gamma^{2}}}^{\mu} d \epsilon \sin \frac{\pi k\left(\epsilon^{2}-\Delta^{2}-\Gamma^{2}\right)}{e B} \exp \left(-\frac{2 \pi k|\epsilon| \Gamma}{e B}\right) \\
& =-\frac{N(e B)^{3 / 2}}{2 \pi} \theta\left(\mu^{2}-\Delta^{2}-\Gamma^{2}\right) \sum_{k=1}^{\infty} \frac{1}{\pi k} \int_{0}^{w} \frac{d x \sin (\pi k x)}{\sqrt{x+u}} \exp (-2 \pi k \sqrt{\gamma(x+u)}),
\end{aligned}
$$

where the variables $w, u$ and $\gamma$ are defined in Eq. (8.6). Using the representation

$$
\frac{e^{-z}}{z}=\frac{1}{2 \sqrt{\pi}} \int_{0}^{\infty} \frac{d t e^{-t-\frac{z^{2}}{4 t}}}{t^{3 / 2}}
$$

we can perform the integration over $x$ to get

$$
\begin{aligned}
& \int_{0}^{w} \frac{d x \sin (\pi k x)}{\sqrt{x+u}} \exp (-2 \pi k \sqrt{\gamma(x+u)}) \\
& =\frac{1}{\pi \sqrt{k}} \int_{0}^{\infty} \frac{d t}{\sqrt{t}\left(t^{2}+1\right)} e^{-\frac{\pi k \gamma}{t}}\left[e^{-t(\pi k u)}-\cos (\pi k w) e^{-t(\pi k v)}-t \sin (\pi k w) e^{-t(\pi k v)}\right]
\end{aligned}
$$

where the variable $v=\mu^{2} / e B$ is the same as in Eq. (6.5). Hence the oscillating part of the thermodynamic potential can be written (omitting the monotonic term related to the first term in square brackets of the last expression) in the final form (8.5) using the functions of two arguments $J_{1}(\mathrm{p}, \mathrm{r})$ and $J_{2}(p, r)$

$$
\sqrt{\pi} J_{1}(p, r)=\int_{0}^{\infty} \frac{d t e^{-t p-\frac{r}{t}}}{\sqrt{t}\left(t^{2}+1\right)}, \quad \sqrt{\pi} J_{2}(p, r)=-\frac{\partial}{\partial p}\left[\sqrt{\pi} J_{1}(p, r)\right]=\int_{0}^{\infty} \frac{d t \sqrt{t} e^{-t p-\frac{r}{t}}}{t^{2}+1} .
$$

As is seen, the functions $J_{1}(p, r), J_{2}(p, r)$ are monotonic functions of their variables.

For large values of the parameter $p$ we can obtain asymptotic expansion of the functions $J_{1}(p, r), J_{2}(p, r)$ changing the variable $t \rightarrow t / p$ and expanding then the denominator in powers of $p$, we get

$$
\sqrt{\pi} J_{1}(p, r)=\sum_{n=0}^{\infty} \frac{(-1)^{n}}{p^{2 n+1 / 2}} \int_{0}^{\infty} \frac{d t e^{-t-\frac{r p}{t}}}{t^{-2 n+1 / 2}}=2 \sum_{n=0}^{\infty}(-1)^{n}\left(\frac{r}{p}\right)^{n+1 / 4} K_{2 n+1 / 2}(2 \sqrt{r p}),
$$

where we used the formula for the representation of the Macdonald function

$$
K_{\nu}(2 z)=\frac{z^{\nu}}{2} \int_{0}^{\infty} \frac{d t e^{-t-\frac{z^{2}}{t}}}{t^{\nu+1}}, \quad \operatorname{Re} z^{2}>0, \quad|\arg z|<\frac{\pi}{2} .
$$

and the relation $K_{-\nu}(z)=K_{\nu}(z)$. Since for half integer values of the index $\nu$

$$
K_{n+\frac{1}{2}}(z)=\sqrt{\frac{\pi}{2 z}} e^{-z} \sum_{k=0}^{n} \frac{(n+k) !}{k !(n-k) !(2 z)^{k}}
$$

(see Eq.(8.468) in [52]), we obtain for the first few terms in the expansion

$$
J_{1}(p, r) \simeq \frac{1}{\sqrt{p}}\left[1-\frac{3}{4 p^{2}}\left(1+2 \sqrt{r p}+\frac{4}{3} r p\right)\right] e^{-2 \sqrt{r p}}, \quad p \rightarrow \infty
$$


Similarly, for $J_{2}$ we get

$$
\begin{aligned}
\sqrt{\pi} J_{2}(p, r) & =2 \sum_{n=0}^{\infty}(-1)^{n}\left(\frac{r}{p}\right)^{n+3 / 4} K_{2 n+3 / 2}(2 \sqrt{r p}) \\
& \simeq \frac{1}{p^{3 / 2}}\left[\frac{1}{2}+\sqrt{r p}-\frac{1}{p^{2}}\left(\frac{15}{8}+\frac{15}{4} \sqrt{r p}+3 r p+(r p)^{3 / 2}\right)\right] e^{-2 \sqrt{r p}}, \quad p \rightarrow \infty .
\end{aligned}
$$

[1] D. Shoenberg, Magnetic oscillations in metals, (Cambridge University Press, Cambridge 1984).

[2] L.D. Landau, Z. Phys. 64, 629 (1930).

[3] J. Singleton, Rep. Progr. Phys. 63, 1111 (2000).

[4] K. Yasui and T. Kita, Phys. Rev. B 66, 184516 (2002).

[5] I.M. Lifshits and A.M. Kosevich, Zh. Eksp. Teor. Fiz. 29, 730 (1955) [Sov. Phys. JETP 2, 636 (1956)].

[6] E. Adams and T. Holstein. J. Phys. Chem. Sol. 10, 254 (1959).

[7] T. Champel and V.P. Mineev, Philosophical Magazine B 81, 55 (2001).

[8] T. Champel, Phys. Rev. B 64, 054407 (2001).

[9] A.A. Abrikosov, Phys. Rev. B 58, 2788 (1998).

[10] B.I. Halperin and T.M. Rice, Solid State Phys. 21, 115 (1968).

[11] J.B. Marston and I. Affleck, Phys. Rev. B 39, 11538 (1989).

[12] A.A. Nersesyan and G.E. Vachanadze, J. Low Temp. Phys. 77, 293 (1989).

[13] C.C. Tsuei and J.R. Kirtley, Rev. Mod. Phys. 72, 969 (2000).

[14] G.W. Semenoff, Phys. Rev. Lett. 53, 2449 (1984).

[15] D.P. Di Vincenzo and E.J. Mele, Phys. Rev. B 29, 1685 (1984).

[16] J. Gonzàlez, F. Guinea, and M.A.H. Vozmediano, Nucl. Phys. B 406, 771 (1993); Phys. Rev. B 63, 134421 (2001).

[17] S. Uji, J.S. Brooks, and Y. Iye, Physica B 246-247, 299 (1998).

[18] Z.M. Wang, Q.Y. Xu, G.Ni, and Y.W. Du, Phys. Lett. A 314, 328 (2003).

[19] Y. Kopelevich, J.H.S. Torres, R.R. da Silva, F. Mrowka, H. Kempa, and P. Esquinazi, Phys. Rev. Lett. 90, 156402 (2003).

[20] Y. Kopelevich, P. Esquinazi, J.H.S. Torres, R.R. da Silva, H. Kempa, F. Mrowka, and R. Ocana, preprint cond-mat/0209442

[21] V.A. Miransky, Progr. Theor. Phys. Suppl. 123, 49 (1996); V.P. Gusynin, Ukr. J. Phys. 45, 603 (2000); W. Dittrich, H. Gies, Probing the Quantum Vacuum: Perturbative Effective action approach in Quantum Electrodynamics and its Application, Springer, 2000.

[22] P. Elmfors, D. Persson, and B.-S. Skagerstam, Phys. Rev. Lett. 71, 480 (1993); Astropart. Phys. 2, 299 (1994).

[23] A.S. Vshivtsev, K.G. Klimenko, and B.V. Magnitskii, Zh. Eksp. Teor. Fiz. 107, 307 (1995) [JETP 80, 162 (1995)].

[24] A.S. Vshivtsev and K. G. Klimenko, Zh. Eksp. Teor. Fiz. 109, 954 (1996) [JETP 82, 514 (1996)].

[25] D. Cangemi and G. Dunne, Ann. Phys. 249, 582 (1996).

[26] T. Inagaki, D. Kimura, and T. Murata, hep-ph/0307289

[27] S. Chakravarty, R.B. Laughlin, D.K. Morr, and C. Nayak, Phys. Rev. B 63, 094503 (2001).

[28] X. Yang and C. Nayak, Phys. Rev. B 65, 064523 (2002).

[29] S.G. Sharapov, V.P. Gusynin, and H. Beck, Phys. Rev. B 67, 144509 (2003).

[30] R. Jackiw and S. Templeton, Phys. Rev. D 23, 2291 (1981).

[31] O. Vafek, A. Melikyan, M. Franz, and Z. Tešanović, Phys. Rev. B 63, 134509 (2001).

[32] J. Ye, Phys. Rev. Lett. 86, 316 (2001).

[33] S.H. Simon and P.A. Lee, Phys. Rev. Lett. 78, 1548 (1997); Phys. Rev. Lett. 78, 5029 (1997); G.E. Volovik, N.B. Kopnin, Phys. Rev. Lett. 78. 5028 (1997).

[34] M. Chiao, R.W. Hill, C. Lupien, L. Taillefer, P. Lambert, R. Gagnon, and P. Fournier, Phys. Rev. B 62, 3554 (2000).

[35] H.K. Nguyen and S. Chakravarty, Phys. Rev. B 65, 180519 (2002).

[36] M. Franz and Z. Tešanović, Phys. Rev. Lett. 84, 554 (2000).

[37] L.P. Gor'kov and J.R. Schrieffer, Phys. Rev. Lett. 80, 3360 (1998).

[38] P.W. Anderson, cond-mat/9812063

[39] N.B. Kopnin and V.M. Vinokur, Phys. Rev. B 62, 9770 (2000).

[40] R. Saito, G. Dresselhaus and M.S. Dresselhaus, Physical Properties of Carbon Nanotubes, Imperial College Press, London, 1998.

[41] D.V. Khveshchenko, Phys. Rev. Lett. 87, 246802 (2001).

[42] E.V. Gorbar, V.P. Gusynin, V.A. Miransky, and I.A. Shovkovy, Phys. Rev. B 66, 045108 (2002).

[43] E.J. Ferrer, V.P. Gusynin, and V. de la Incera, Eur. Phys. J. B 33, 397 (2003).

[44] V.P. Gusynin, V.A. Miransky, and I.A. Shovkovy, Phys. Rev. Lett. 73, 3499 (1994); Phys. Rev. D 52, 4718 (1995).

[45] A. Chodos, K.Everding, and D.A. Owen, Phys. Rev. D 42, 2881 (1990).

[46] R.E. Prange, The Quantum Hall Effect, edited by R.E. Prange and S.M. Girvin, (Springer Verlag, New York, 1987).

[47] T. Champel, V. Mineev, Phys. Rev. B 66, 195111 (2002); 67, 089901 (E) (2003).

[48] D. Shoenberg, J. Low Temp. Phys. 56, 417 (1984).

[49] A.C. Durst and P.A. Lee, Phys. Rev. B 62, 1270 (2000). 
[50] P. Grigoriev, Zh. Eksp. Teor. Fiz. 119, 1257 (2001) [JETP 92, 1090 (2001)].

[51] A.S. Alexandrov, A.M. Bratkovsky, Phys. Rev. Lett. 76, 1308 (1996).

[52] I.S. Gradshtein and I.M. Ryzhik, Table of Integrals, Series and Products, (Academic Press, Orlando, 1980).

[53] The functions $J_{1}, J_{2}$ are related to the functions $P, Q$ used in Ref. 24] as follows $J_{1}(x)=\sqrt{x} P(x), J_{2}(x)=-\sqrt{x} Q(x)$. 


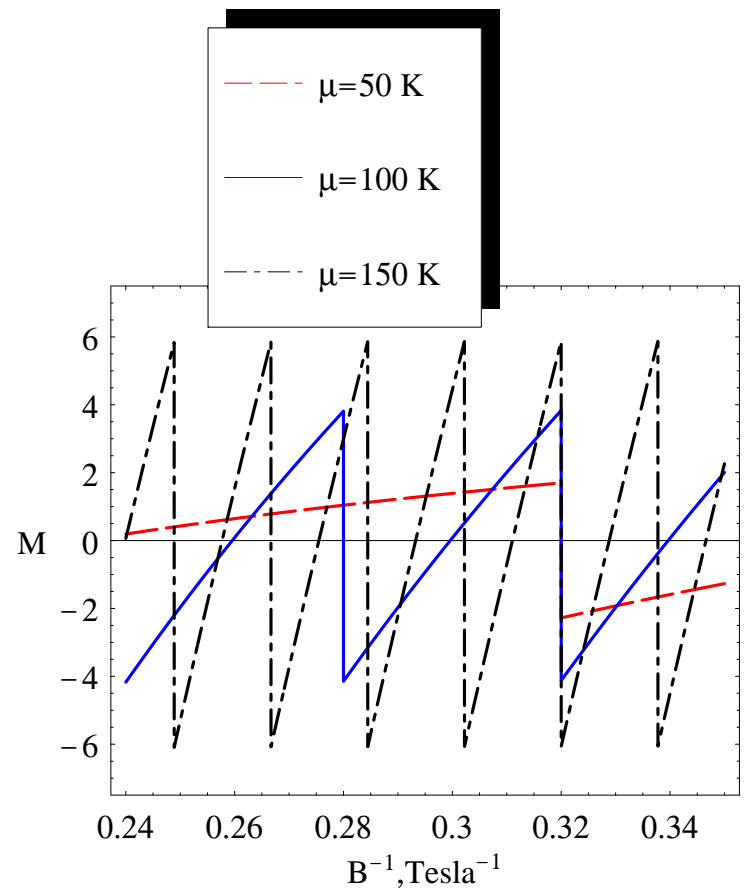

FIG. 1: The magnetization $M$ (in $\mathrm{K} /\left(\right.$ Tesla $\left.\mathrm{cm}^{2}\right)$ calculated for $N=1$ ) as a function of inverse field, $B^{-1}$, for three different values of chemical potential and $\Delta=T=\Gamma=0$. We use $e B \rightarrow 200 \mathrm{~K}^{2} \cdot B$ [Tesla].

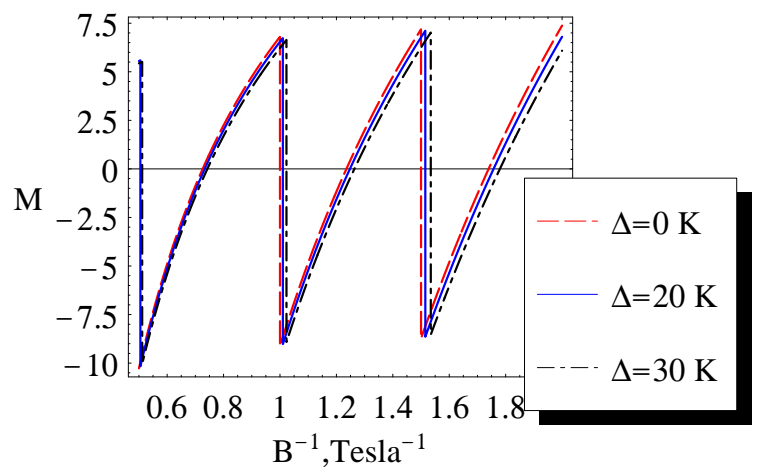

FIG. 2: The magnetization $M$ (in $\mathrm{K} /\left(\right.$ Tesla $\left.\mathrm{cm}^{2}\right)$ calculated for $N=1$ ) as a function of inverse field, $B^{-1}$, for three different values of the gap $\Delta$ and for $T=\Gamma=0$. We use $e B \rightarrow 10^{4} \mathrm{~K}^{2} \cdot B$ [Tesla] and $\mu=200 \mathrm{~K}$ that are typical for graphite. 


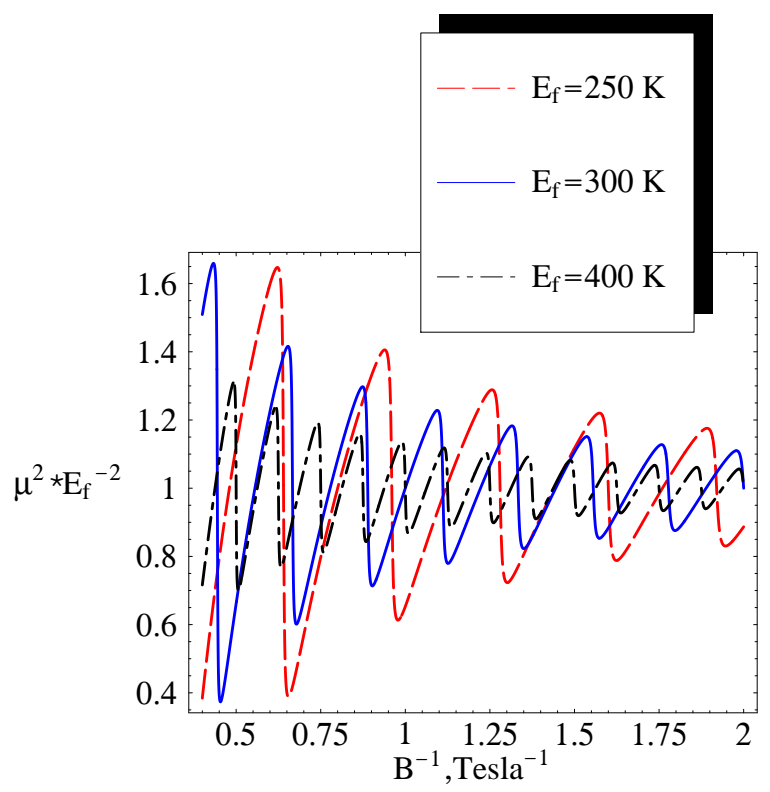

FIG. 3: The chemical potential $\left(\mu / \epsilon_{F}\right)^{2}$ as a function of inverse field, $B^{-1}$, for three different values of the Fermi energy $\epsilon_{F}$, $\Gamma=0.5 \mathrm{~K}$ and $T=\Delta=0$. We use $e B \rightarrow 10^{4} \mathrm{~K}^{2} \cdot B$ [Tesla $]$.

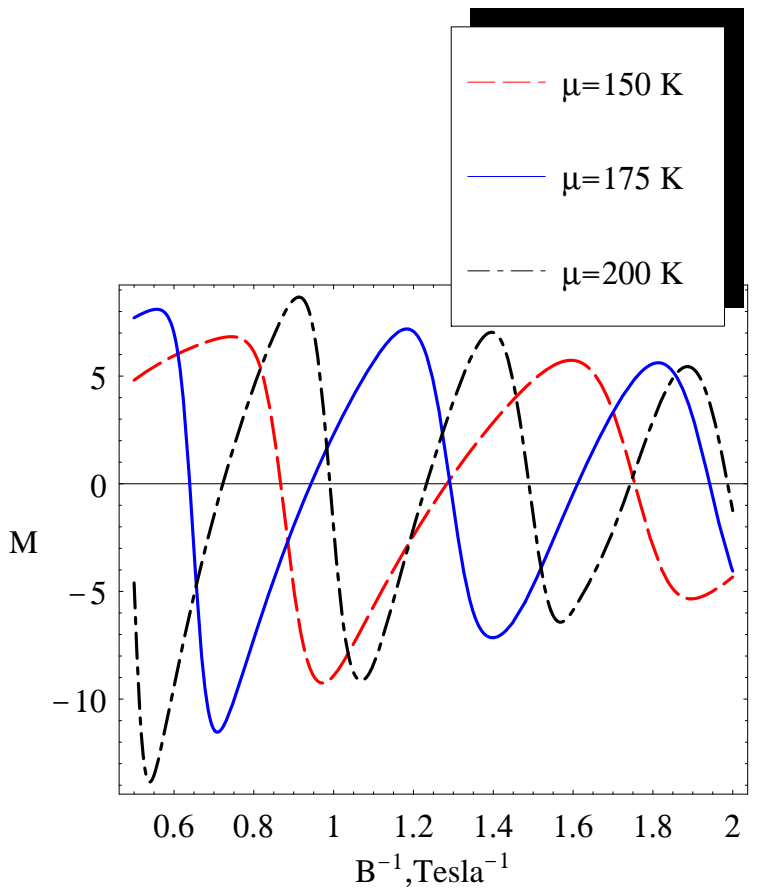

FIG. 4: The magnetization $M$ (in $\mathrm{K} /\left(\right.$ Tesla $\left.\mathrm{cm}^{2}\right)$ calculated for $N=1$ ) as a function of inverse field, $B^{-1}$, for three different values of the chemical potential. We use $e B \rightarrow 10^{4} \mathrm{~K}^{2} \cdot B$ [Tesla], $\Gamma=0.5 \mathrm{~K}, T=5 \mathrm{~K}$ and $\Delta=0$. 


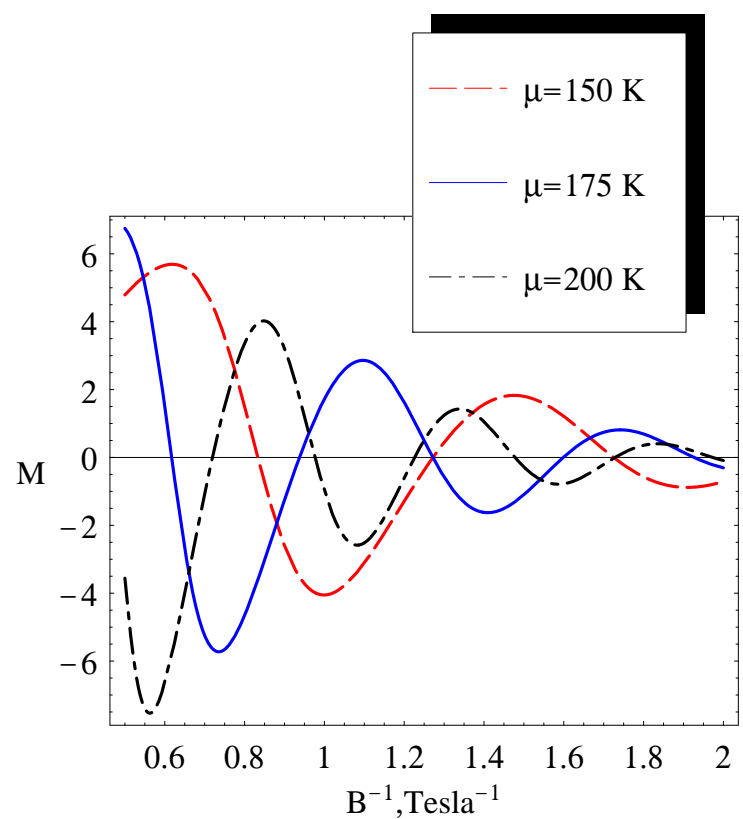

FIG. 5: The magnetization $M$ (in $\mathrm{K} /\left(\right.$ Tesla $\left.\mathrm{cm}^{2}\right)$ calculated for $N=1$ ) as a function of inverse field, $B^{-1}$, for three different values of the chemical potential. We use $e B \rightarrow 10^{4} \mathrm{~K}^{2} \cdot B$ [Tesla], $\Gamma=0.5 \mathrm{~K}, T=15 \mathrm{~K}$ and $\Delta=0$. 